\title{
RUNX2 regulates leukemic cell metabolism and chemotaxis in high-risk T cell acute lymphoblastic leukemia
}

Filip Matthijssens, ${ }^{1,2}$ Nitesh D. Sharma, ${ }^{3,4}$ Monique Nysus, ${ }^{3,4}$ Christian K. NickI, ${ }^{3,4}$ Huining Kang, ${ }^{4,5}$ Dominique R. Perez, ${ }^{4,6}$ Beatrice Lintermans, ${ }^{1,2}$ Wouter Van Loocke, ${ }^{1,2}$ Juliette Roels, ${ }^{1,2}$ Sofie Peirs, ${ }^{1,2}$ Lisa Demoen, ${ }^{1,2}$ Tim Pieters, ${ }^{1,2}$ Lindy Reunes, ${ }^{1,2}$ Tim Lammens, ${ }^{2,7}$ Barbara De Moerloose, ${ }^{2,7}$ Filip Van Nieuwerburgh, ${ }^{8}$ Dieter L. Deforce, ${ }^{8}$ Laurence C. Cheung, ${ }^{9,10}$ Rishi S. Kotecha, ${ }^{9,10}$ Martijn D.P. Risseeuw, ${ }^{2,11}$ Serge Van Calenbergh, ${ }^{2,11}$ Takeshi Takarada, ${ }^{12}$ Yukio Yoneda, ${ }^{13}$ Frederik W. van Delft, ${ }^{14}$ Richard B. Lock, ${ }^{15}$ Seth D. Merkley, ${ }^{5}$ Alexandre Chigaev, ${ }^{4,6}$ Larry A. Sklar, ${ }^{4,6}$ Charles G. Mullighan, ${ }^{16}$ Mignon L. Loh, ${ }^{17}$ Stuart S. Winter, ${ }^{18}$ Stephen P. Hunger, ${ }^{19}$ Steven Goossens, ${ }^{1,2,20}$ Eliseo F. Castillo, ${ }^{5}$ Wojciech Ornatowski, ${ }^{21}$ Pieter Van Vlierberghe, ${ }^{1,2}$ and Ksenia Matlawska-Wasowska3,4

'Department of Biomolecular Medicine, Ghent University, Chent, Belgium. ${ }^{2}$ Cancer Research Institute Chent (CRIC), Ghent, Belgium. ${ }^{3}$ Department of Pediatrics, Division of Hematology-Oncology, University of New Mexico Health Sciences Center, Albuquerque, New Mexico, USA. ${ }^{4}$ Comprehensive Cancer Center, University of New Mexico, Albuquerque, New Mexico, USA. ${ }^{5}$ Department of Internal Medicine, University of New Mexico Health Sciences Center, Albuquerque, New Mexico, USA. ${ }^{6}$ University of New Mexico Center for Molecular Discovery, Albuquerque, New Mexico, USA. 'Department of Pediatric HematologyOncology and Stem Cell Transplantation, Chent University Hospital, Chent, Belgium. ' ${ }^{\circ}$ Laboratory of Pharmaceutical Biotechnology, Ghent University, Ghent, Belgium. ${ }^{9}$ Telethon Kids Cancer Centre, Telethon Kids Institute, University of Western Australia, Perth, Western Australia, Australia. ${ }^{10 S}$ chool of Pharmacy and Biomedical Sciences, Curtin University, Perth, Western Australia, Australia. "Laboratory for Medicinal Chemistry, Ghent University, Ghent, Belgium. '²Department of Regenerative Science, Okayama University Graduate School of Medicine, Dentistry and Pharmaceutical Sciences, Okayama, Japan. ${ }^{13}$ Department of Pharmacology, Osaka University Graduate School of Dentistry, Suita, Japan. ${ }^{14}$ Wolfson Childhood Cancer Research Centre, Newcastle University Centre for Cancer, Newcastle upon Tyne, United Kingdom. ${ }^{15}$ Children's Cancer Institute, School of Women's and Children's Health, Lowy Cancer Centre, University of New South Wales, Sydney, New South Wales, Australia. ${ }^{16} \mathrm{Department}$ of Pathology, St. Jude Children's Research Hospital, Memphis, Tennessee, USA. " Department of Pediatrics, Benioff Children's Hospital, UCSF, San Francisco, California, USA. ${ }^{18}{ }^{2}$ ancer and Blood Disorders Program, Children's Minnesota, Minneapolis, Minnesota, USA. ${ }^{19}$ Department of Pediatrics and the Center for Childhood Cancer Research, Children's Hospital of Philadelphia and the Perelman School of Medicine at the University of Pennsylvania, Philadelphia, Pennsylvania, USA. ${ }^{20}$ Department of Diagnostic Sciences, Ghent University, Ghent, Belgium. ${ }^{21}$ Department of Medicine, University of Arizona, Tucson, Arizona, USA.

T cell acute lymphoblastic leukemia (T-ALL) is an aggressive hematologic malignancy with inferior outcome compared with that of B cell ALL. Here, we show that Runt-related transcription factor 2 (RUNX2) was upregulated in high-risk T-ALL with $K M T 2 A$ rearrangements (KMT2A-R) or an immature immunophenotype. In KMT2A-R cells, we identified $R U N X 2$ as a direct target of the $K M T 2 A$ chimeras, where it reciprocally bound the $K M T 2 A$ promoter, establishing a regulatory feed-forward mechanism. Notably, RUNX2 was required for survival of immature and KMT2A-R T-ALL cells in vitro and in vivo. We report direct transcriptional regulation of CXCR4 signaling by RUNX2, thereby promoting chemotaxis, adhesion, and homing to medullary and extramedullary sites. RUNX2 enabled these energy-demanding processes by increasing metabolic activity in T-ALL cells through positive regulation of both glycolysis and oxidative phosphorylation. Concurrently, RUNX2 upregulation increased mitochondrial dynamics and biogenesis in T-ALL cells. Finally, as a proof of concept, we demonstrate that immature and KMT2A-R T-ALL cells were vulnerable to pharmacological targeting of the interaction between RUNX2 and its cofactor CBF $\beta$. In conclusion, we show that RUNX2 acts as a dependency factor in high-risk subtypes of human T-ALL through concomitant regulation of tumor metabolism and leukemic cell migration.

\section{Introduction}

$\mathrm{T}$ cell acute lymphoblastic leukemia (T-ALL) is an aggressive hematologic malignancy that occurs predominantly in children, adolescents, and young adults. It is currently treated by multia-

Authorship note: FM and NDS contributed equally to this work. PVV and KMW are co-senior authors.

Conflict of interest: The authors have declared that no conflict of interest exists. Copyright: (c) 2021, American Society for Clinical Investigation.

Submitted: June 22, 2020; Accepted: January 20, 2021; Published: March 15, 2021 Reference information: J Clin Invest. 2021;131(6):e141566.

https://doi.org/10.1172/JCl141566. gent high-dose chemotherapy that is often associated with acute and chronic life-threatening and debilitating toxicities $(1,2)$.

T-ALL can be classified by unique gene expression signatures corresponding to different stages of $\mathrm{T}$ cell developmental arrest and showing aberrant regulation of specific transcription factor oncogenes, including LMO2/LYL1, HOXA, TLX1, TLX3, NKX2.1, NKX2.2, TAL1, TAL2, or LMO1/2 (3-8). A high-risk subtype of T-ALL derived from cells at the early $\mathrm{T}$ cell precursor (ETP) differentiation stage significantly overlaps with $L Y L 1$-positive T-ALL (4) and MEF2C-dysregulated immature T-ALL (5, 9). These immature $\mathrm{T}$ cell leukemias show a transcriptional program related to hematopoietic stem cells and myeloid progenitors (5) and are 
associated with a poor prognosis, with reduced overall survival (10-12). Deregulated HOXA gene expression is a hallmark of KMT2A-rearranged (KMT2A-R) T cell leukemia $(6,13,14)$. Chromosomal rearrangements involving the KMT2A $(M L L)$ gene are considered a poor prognostic factor in all leukemia subtypes (15), including T-ALL $(16,17)$. Other genetic alterations that result in HOXA overexpression in T-ALL include the PICALM-AF1O and SET-NUP214 fusion genes (16).

The RUNX family of heterodimeric transcription factors consists of 3 members, Runt-related transcription factor 1 (RUNX1), RUNX2, and RUNX3, which have crucial roles in the regulation of normal development and tumorigenesis $(18,19)$. All 3 RUNX family members share an evolutionarily conserved Runt domain, which is required for binding with both DNA and the non-DNAbinding protein $\mathrm{CBF} \beta$. Interaction of RUNX-CBF $\beta$ increases DNA-binding affinity of the heterodimeric complex and is crucial for its transactivation function (20). While RUNX1 is predominantly expressed in the hematopoietic lineage and plays a crucial role in embryonic and adult hematopoiesis $(21,22)$, RUNX2 has been mainly characterized as a pivotal regulator for osteoblast and chondrocyte differentiation and bone development (23-26).

Few studies have investigated the role of RUNX2 in normal hematopoiesis and T cell development. Runx 2 is highly expressed in the hematopoietic stem cell and progenitor compartment of the $\mathrm{BM}$ in mice, and its upregulation leads to repression of myeloid differentiation (27). Another study in mice found that Runx2 is mainly expressed in the CD4/CD8 double-negative T cell population and that its enforced expression perturbs $\mathrm{T}$ cell development at the $\beta$-selection stage (28).

To date, mainly oncogenic roles have been described for RUNX2 in tumor development. Amplification of RUNX2 has been implicated in osteosarcoma development and chemoresistance (29-31). High RUNX2 expression levels have also been reported in breast and prostate cancer, most prominently in the context of bone metastasis $(32,33)$. In hematologic malignancies, mouse models demonstrated that Runx 2 cooperates with Cbf $\beta$-SMMHC to induce AML (27). Furthermore, Runx2 was identified as a retroviral insertion site in retrovirus-induced hematopoietic tumors (34), and its oncogenic potential was further established in CD2Run $x 2$ transgenic mice (35). CD2-Runx 2 transgenic mice are prone to $\mathrm{T}$ cell lymphoma development where RUNX2 collaborates with MYC to overcome apoptosis and growth arrest (36). In blastic plasmacytoid dendritic cell neoplasm, RUNX2 is also highly expressed and collaborates with MYC to promote tumor growth (37).

Given the roles of RUNX2 in tumor development and metastasis, we investigated its roles in the pathobiology of T-ALL. We found that increased RUNX2 levels are largely restricted to immature and KMT2A-R T-ALL and that RUNX2 acts as a dependency factor in these high-risk T-ALL subtypes. RUNX2 is required for T-ALL survival, and its aberrant expression enhances disease progression and dissemination to extramedullary sites. Functionally, RUNX2 induces CXCR4-mediated T-ALL cell migration and increases leukemic cell metabolism by positive regulation of both glycolysis and mitochondrial oxidative phosphorylation. Consequently, immature and KMT2A-R T-ALL are vulnerable to pharmacological targeting of the RUNX-CBF $\beta$ interaction.

\section{Results}

RUNX2 is highly expressed in immature and KMT2A-rearranged $T$-ALL. First, we evaluated RUNX2 expression in different molecular subtypes of T-ALL. We analyzed RUNX2 expression in RNASeq data sets of human T-ALL classified into subgroups with deregulation of the following genes: TAL1 $(n=87)$, TAL2 $(n=8)$, LMO1/2 $(n=10), T L X 1(n=26), T L X 3(n=46)$, NKX2 $(n=14)$, HOXA ( $n=33)$, and LYL1 $(n=18)$ (Figure 1A and ref. 8). RUNX2 displayed significantly $(P<0.05)$ higher expression levels in HOXA and LYL1 subtypes compared with other T-ALL subtypes. While ETP or immature T-ALL commonly show LYL1 deregulation, chromosomal alterations involving the $K M T 2 A$ gene are hallmarks of HOXA-deregulated leukemia. Within the HOXA group, RUNX2 expression was markedly upregulated in samples harboring KMT2A-R compared with the remaining cases (Figure 1B). In addition, patients with ETP (8) showed significantly higher levels of RUNX2 mRNA compared with near-ETP and non-ETP subgroups (Figure 1C). We validated our findings in 2 independent microarray data sets $(16,38)$ and confirmed increased expression of RUNX2 in samples with KMT2A-R (Supplemental Figure 1A; supplemental material available online with this article; https:// doi.org/10.1172/JCI141566DS1). Similarly, samples defined as ETP-ALL by flow cytometry or as immature by gene-expression profiling also exhibited higher levels of RUNX2 mRNA (Supplemental Figure 1, B and C).

Next, we analyzed RUNX2 expression in human T-ALL cell lines, including the KMT2A-FOXO4 rearranged T-ALL cell line KARPAS-45, the immature/ETP phenotype T-ALL cell lines LOUCY and PER-117, and a panel of 6 other T-ALL cell lines. Quantitative reverse-transcriptase PCR (qRT-PCR) and Western blot confirmed that KARPAS-45, PER-117, and LOUCY showed the highest levels of RUNX2 expression across the tested cell lines (Figure 1, D and E).

To further validate our findings, we quantified RUNX2 mRNA levels in primary T-ALL patient samples obtained from the Children's Oncology Group study AALLO434 (2) and the University of New Mexico $(n=25)$ or Ghent University Hospital $(n=11)$. T-ALL samples with an immature (pro-T or pre-T) phenotype showed the highest levels of RUNX2 mRNA along with 1 sample with a cortical immunophenotype and a KMT2A-MLLT1 translocation (Figure $1 \mathrm{~F})$. We also confirmed that samples from T-ALL KMT2A-R patients expressed higher levels of RUNX2 mRNA and protein compared with samples without such lesions (Figure 1, G and $\mathrm{H}$ ). In addition, we performed RUNX2 protein quantification using immunostaining flow cytometry on a panel of 14 patient-derived xenograft (PDX) samples. As expected, immature and KMT2A-R samples presented with the highest protein levels of RUNX2 (Figure 1I). Collectively, these results indicate that RUNX2 is upregulated in ETP/LYL1 ${ }^{+}$/immature and KMT2A-R T-ALL.

Runx2 ablation does not largely affect normal hematopoiesis or $T$ cell development. Since the gene expression signatures of molecular T-ALL subgroups are closely related to those of normal thymocytes at distinct stages of $\mathrm{T}$ cell development (4, $10)$, we analyzed to determine whether the elevated RUNX2 levels in immature T-ALL cells are also reflected in immature normal $\mathrm{T}$ cells. Analysis of different $\mathrm{T}$ cell subsets showed that RUNX2 mRNA levels were highest in CD $34^{+} \mathrm{T}$ cells and steeply 

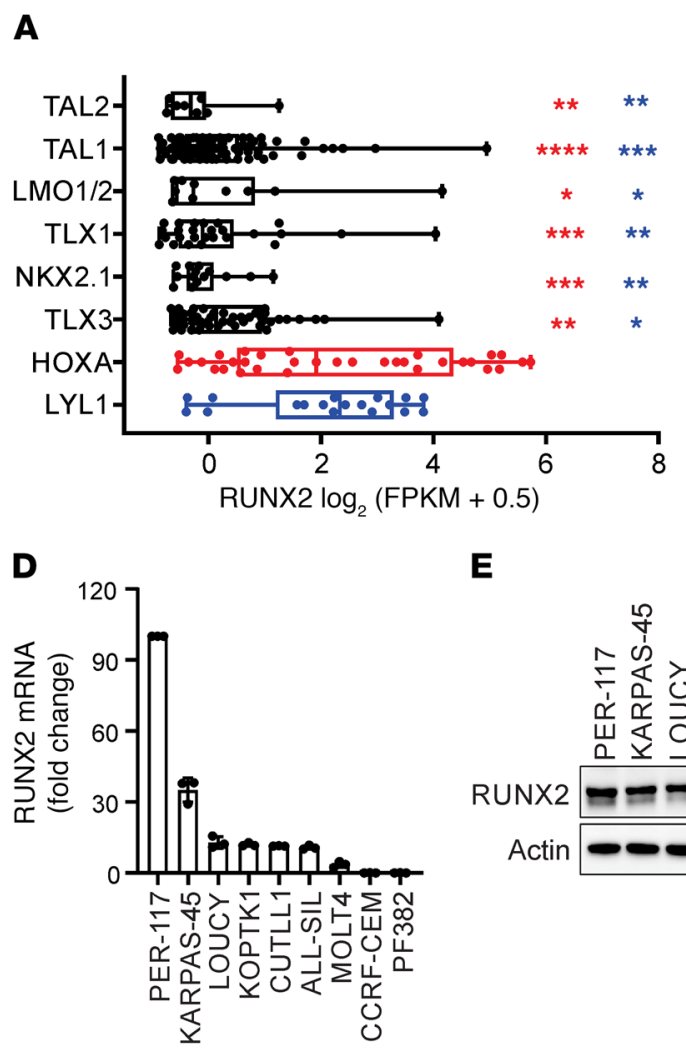

$\mathbf{E}$
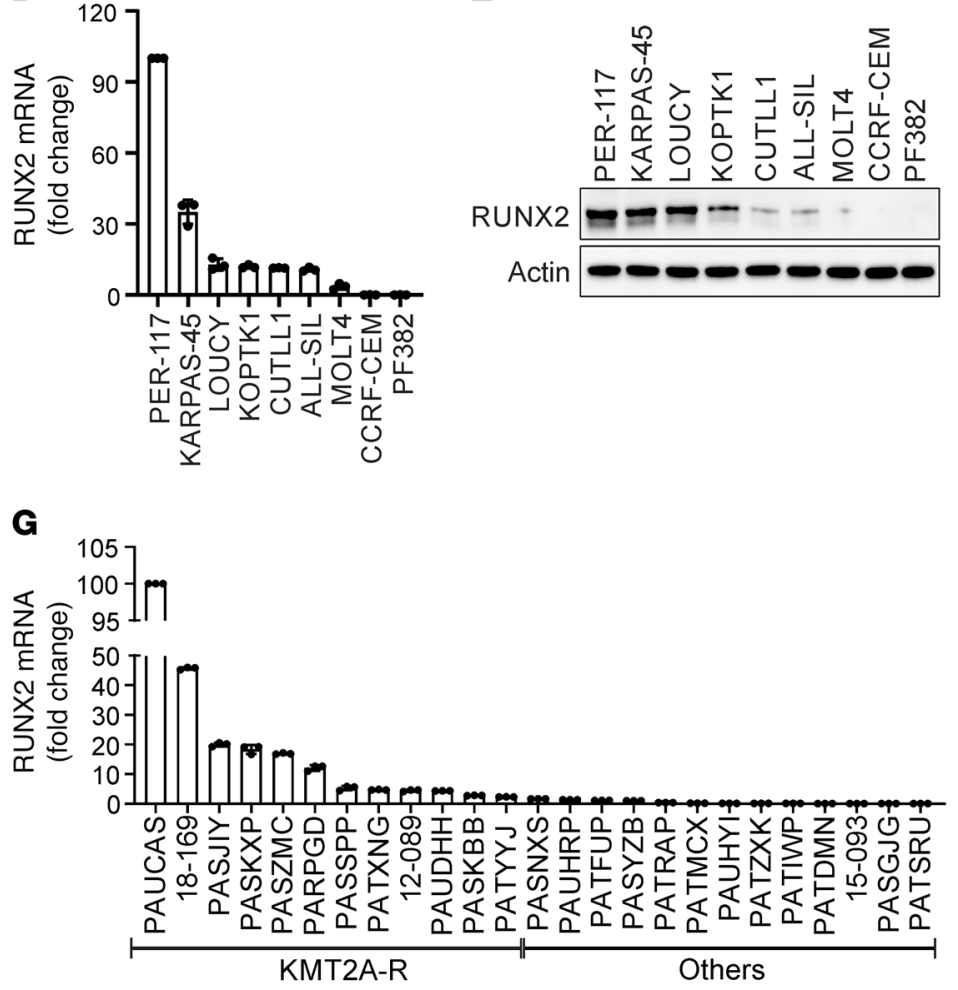
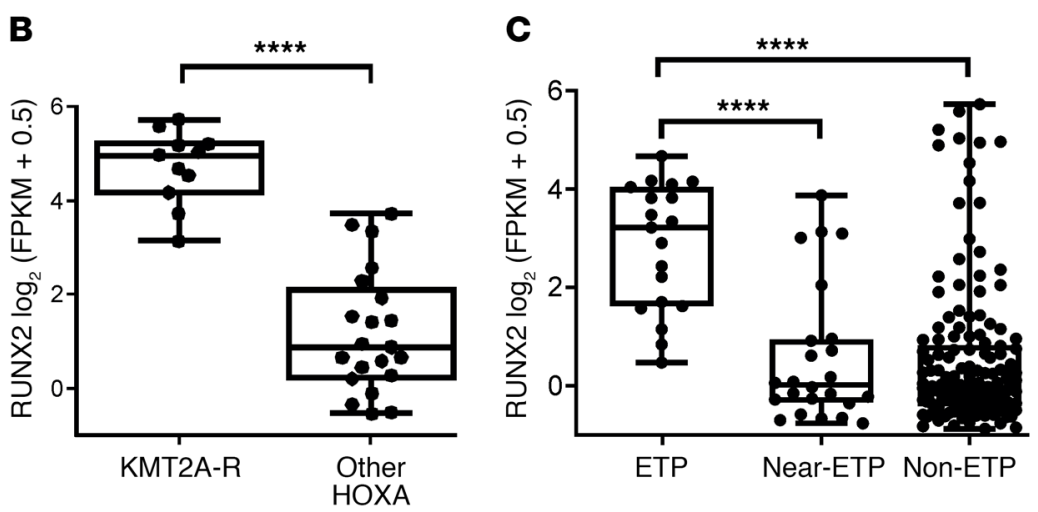

$\mathbf{F}$
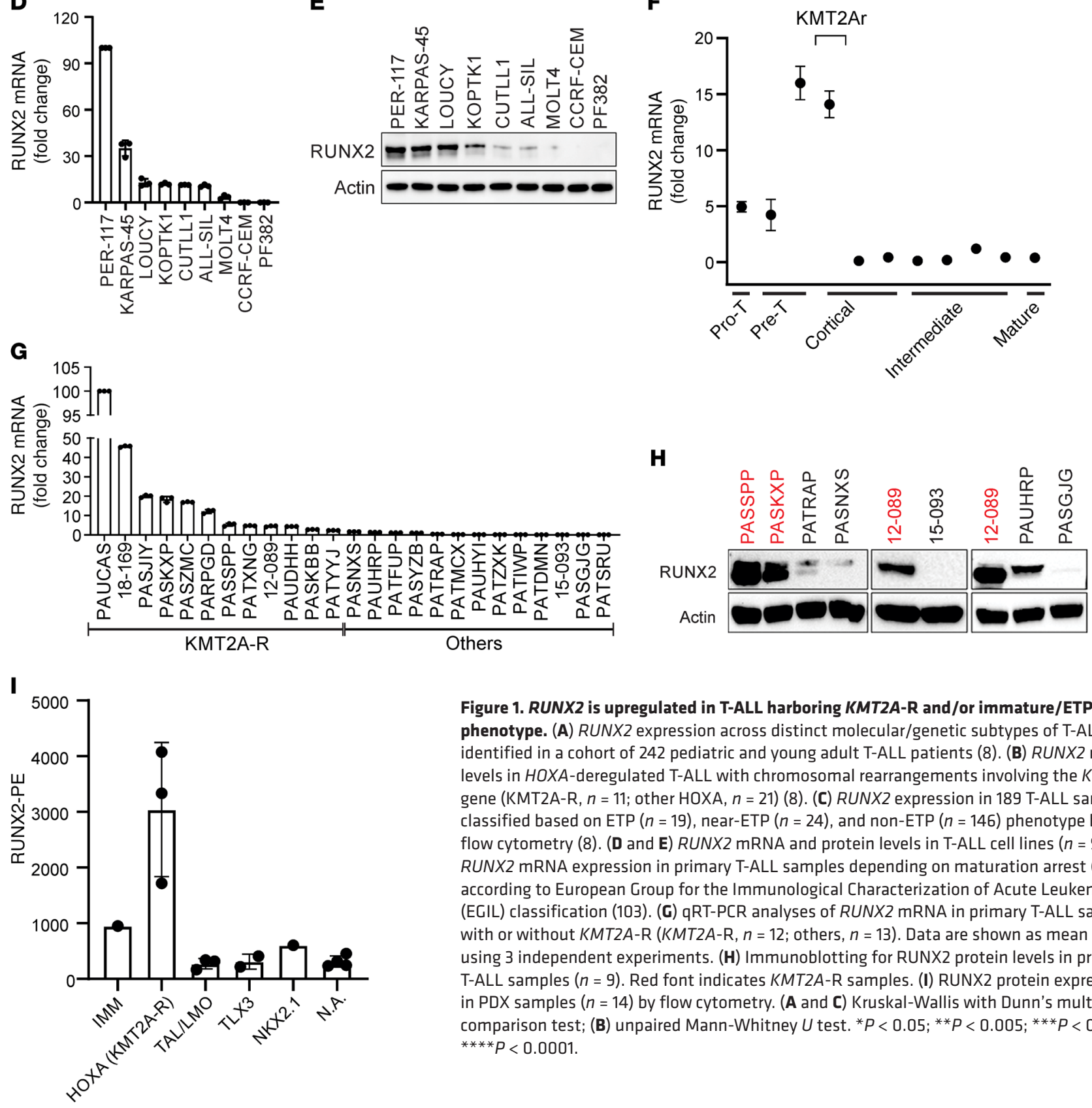

Figure 1. RUNX2 is upregulated in T-ALL harboring KMT2A-R and/or immature/ETP phenotype. (A) RUNX2 expression across distinct molecular/genetic subtypes of T-ALL identified in a cohort of 242 pediatric and young adult T-ALL patients (8). (B) RUNX2 mRNA levels in HOXA-deregulated T-ALL with chromosomal rearrangements involving the KMT2A gene (KMT2A-R, $n=11$; other HOXA, $n=21$ ) (8). (C) RUNX2 expression in 189 T-ALL samples classified based on ETP $(n=19)$, near-ETP $(n=24)$, and non-ETP $(n=146)$ phenotype by flow cytometry (8). (D and E) RUNX2 mRNA and protein levels in T-ALL cell lines $(n=9)$. (F) $R U N X 2$ mRNA expression in primary T-ALL samples depending on maturation arrest $(n=11)$ according to European Group for the Immunological Characterization of Acute Leukemias (EGIL) classification (103). (G) qRT-PCR analyses of RUNX2 mRNA in primary T-ALL samples with or without $K M T 2 A-\mathrm{R}(K M T 2 A-\mathrm{R}, n=12$; others, $n=13)$. Data are shown as mean $\pm \mathrm{SD}$, using 3 independent experiments. (H) Immunoblotting for RUNX2 protein levels in primary T-ALL samples $(n=9)$. Red font indicates KMT2A-R samples. (I) RUNX2 protein expression in PDX samples $(n=14)$ by flow cytometry. (A and $\mathbf{C}$ ) Kruskal-Wallis with Dunn's multiple comparison test; (B) unpaired Mann-Whitney $U$ test. ${ }^{*} P<0.05$; ${ }^{* *} P<0.005$; ${ }^{* * *} P<0.0005$; ${ }^{* * * * P}<0.0001$. 
A

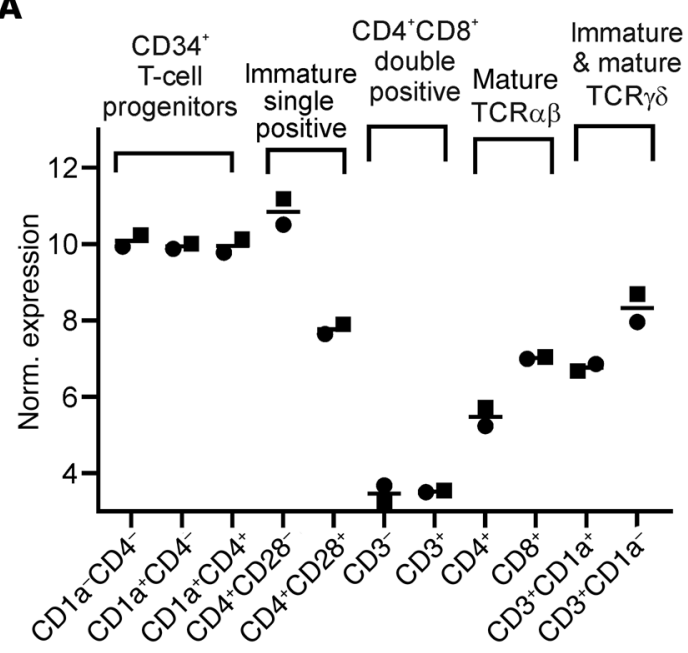

D

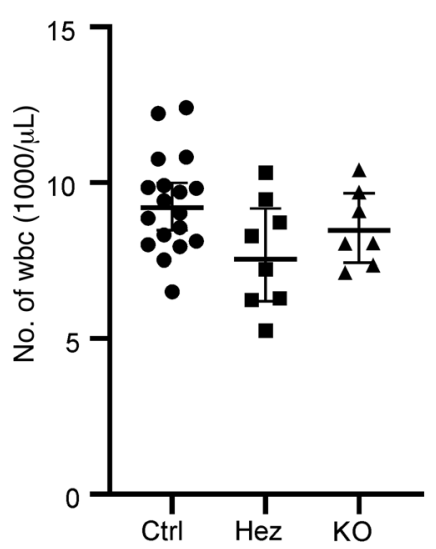

G

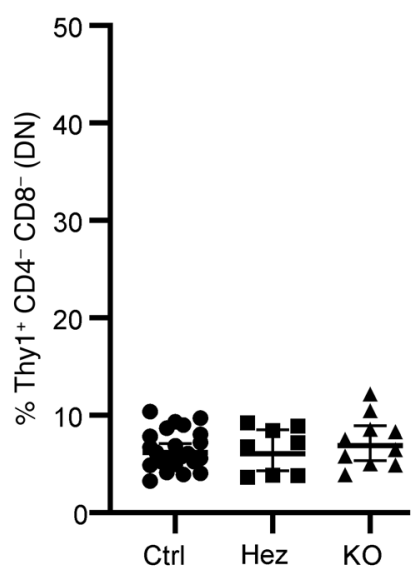

H
E

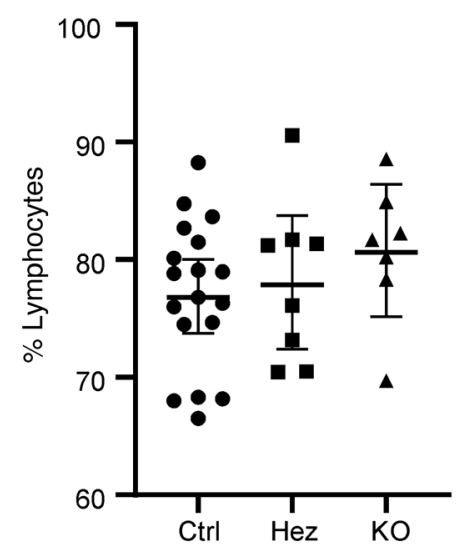

B

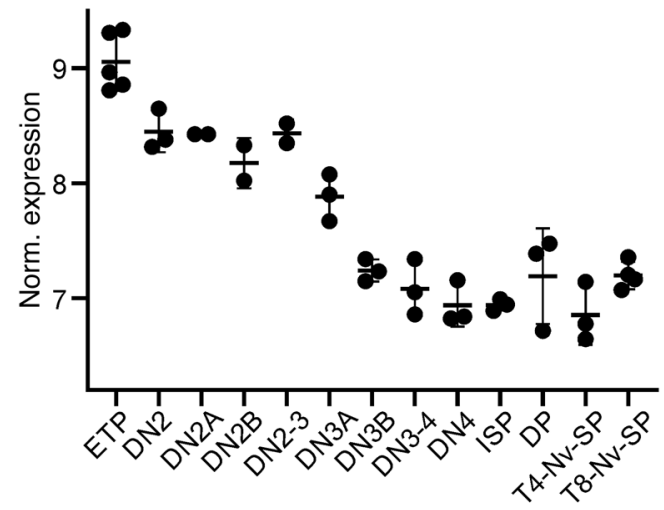

$\mathbf{F}$
C

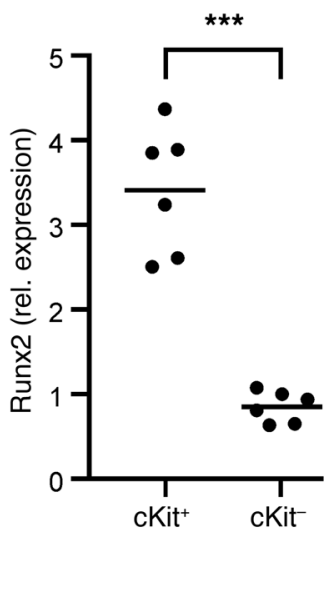

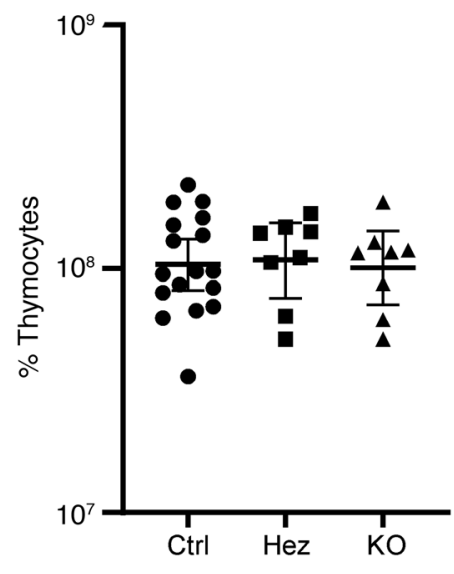

I

J
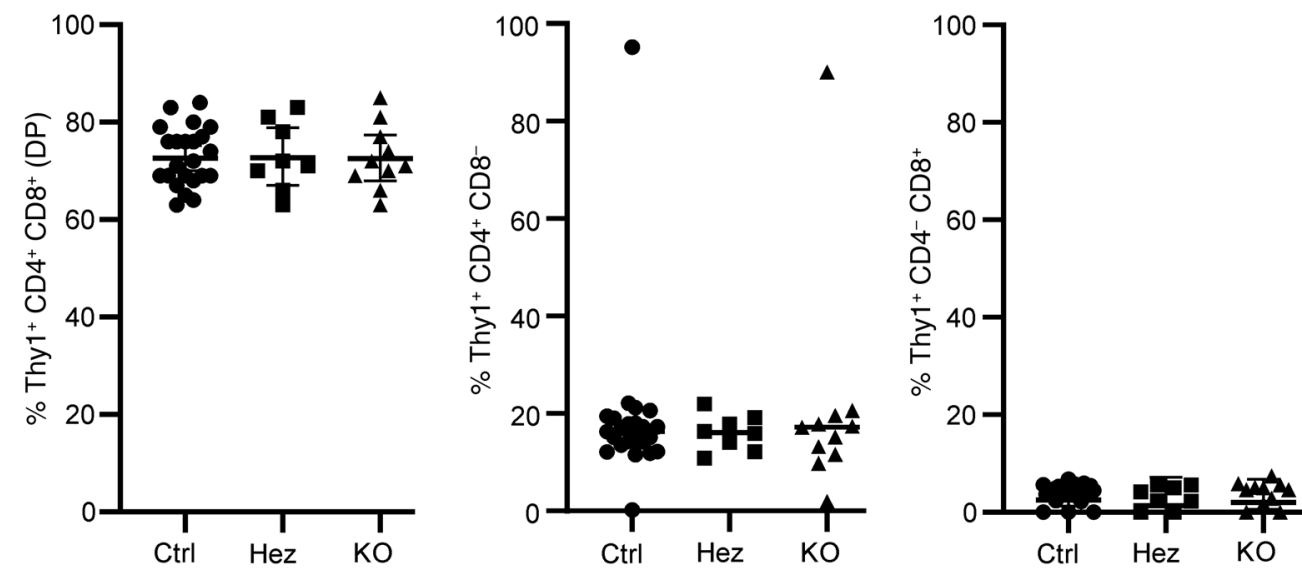

Figure 2. RUNX2 is highly expressed in ETPs, but has no major impact on phenotypic markers of normal hematopoiesis or T cell development. RUNX2 expression in human (A) and mouse (B) T cell subsets. (C) qRT-PCR for Runx2 expression in cKit-enriched and cKit-negative mouse thymocytes. Rel.,

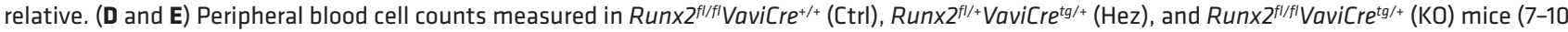
weeks). (F) Total cell number extracted from thymuses. (G-J) Percentages of T cell subsets measured by flow cytometry. (G) DP, double-negative T cells, (H) DN, double-positive T cells, (I) CD4 single-positive T cells, (J) CD8 single-positive T cells. (C) Paired 2-tailed $t$ test; (D-J) 1-way ANOVA with Tukey's multiple comparison test. ${ }^{* *} P<0.0005$. 
decreased toward the $\mathrm{CD} 4^{+} \mathrm{CD} 8^{+}$double-positive stage (Figure $2 \mathrm{~A})$. In mouse $\mathrm{T}$ cell subsets, Runx2 expression was highest in ETP cells and gradually decreased over the different maturation stages (Figure 2B), corresponding with findings in a previous study (28). qRT-PCR in cKit-enriched thymocytes confirmed elevated Runx2 expression levels compared with those in mature, cKit-negative fraction (Figure 2C).

To explore whether Runx 2 is crucial for normal hematopoiesis and T cell development, we established a Vav-iCre-Runx2 KO mouse model (Supplemental Figure 2, A and B, and ref. 39). Both mice harboring homozygous deletion of Runx2 in the hematopoietic system (Runx $2^{f l / f l} \mathrm{Vav}-i \mathrm{Cr} e^{t g /+}$ ) and heterozygous mice (Runx$\left.2^{f /+} \mathrm{Vav}-i \mathrm{Cr} e^{t g /+}\right)$ were viable, fertile, and phenotypically normal (not shown). To examine the effects of RUNX2 on steady-state hematopoiesis, peripheral blood counts were performed on a panel of control ( $R u n x 2^{f / f l} \mathrm{Vav}-i \mathrm{Cr} e^{+/+}$), heterozygous, and homozygous Runx2 KO mice of 6 to 10 weeks of age. No significant differences were observed in WBC, lymphocyte, neutrophil, RBC, hemoglobin or platelet counts (Figure 2, D and E, and Supplemental Figure 2, C-F). A comparison of thymocyte number and T cell populations at different stages of maturation did not show significant differences (Figure 2, F-J, and Supplemental Figure 2, G-L). While Runx2 upregulation in ETPs may suggest its role during early $\mathrm{T}$ cell development, no effect could be identified upon Runx2 deletion on the main $\mathrm{T}$ cell subsets or major hematopoietic processes. Further studies are warranted to determine the roles of RUNX2 in hematopoietic stem and progenitor cells.

RUNX2 is required for the survival of immature and KMT2Arearranged $T-A L L$. With evidence that RUNX2 is upregulated in high-risk leukemias, we investigated its role in growth and survival on KMT2A-R and immature T-ALL cells. We performed shRNAmediated knockdown of RUNX2 in the KMT2A-R cell line KARPAS45 and the immature/ETP-phenotype cell line LOUCY (Supplemental Figure 3, A and B). We found that reduced levels of RUNX2 impaired the proliferation and survival of both cell lines (Figure 3A).

Inactivation of RUNX2 expression in KARPAS- 45 and LOUCY increased the number of early apoptotic cells and delayed cell cycle progression in $S$ and $G_{2} / M$ phases while increasing that in $\mathrm{G}_{1} / \mathrm{G}_{0}$ (Figure 3, B and C, and Supplemental Figure 3C). Functionally, RUNX2 silencing reduced the activation of prosurvival AKT and ERK1/2 pathways (Figure 3D). While RUNX2 depletion had no effect on p53 and BCL2, we found a significant decrease in the levels of active $\beta$-catenin and survivin, which are known downstream targets of AKT signaling (40-42) (Figure 3D). Next, we silenced RUNX2 in 2 primary KMT2A-R samples and confirmed that RUNX2 depletion reduced phosphorylated AKT and ERK1/2, active $\beta$-catenin, and survivin (Figure 3D). To explore the functional link between KMT2A-R and RUNX2, we knocked down RUNX2 in MOHITO and Ba/F3 cells transduced with KMT2AMLLT1 or KMT2A-MLLT4 (Figure 3D and Supplemental Figure $3, \mathrm{D}-\mathrm{F})$. Forced expression of KMT2A-R led to activation of AKT and ERK1/2 pathways and induced upregulation of $\beta$-catenin and survivin. As expected, RUNX2 depletion reduced levels of the tested proteins, suggesting its role in mediating survival signaling in KMT2A-R cells. We next confirmed that apoptotic cell death in RUNX2-depleted cells was associated with cleaved caspase-3, -7 , and -9 (Figure 3D and Supplemental Figure 3F). In our ChIP-
Seq data (described in the next paragraph), we identified RUNX2 binding at the CTNNB1 and BIRC5 promoters (Figure 3E) and at an enhancer region upstream of CTNNB1. Combined with the observation that RUNX2 silencing in KARPAS-45 and LOUCY cells significantly reduced CTNNB1 and BIRC5 mRNA levels, this suggests a direct regulatory role by RUNX2 (Supplemental Figure $3 G)$. Importantly, overexpression of active $\beta$-catenin or survivin partially rescued LOUCY cells from RUNX2 depletion-induced cell death (Figure 3, F-I, and Supplemental Figure 3, H and I). Collectively, these results indicate that RUNX2 regulates cell survival in ETP/immature and KMT2A-R leukemic cells in vitro.

We next investigated to determine whether RUNX2 regulates T-ALL engraftment and disease progression in vivo. Immunodeficient NOD.Cg-Prkd $c^{\text {scid }} I l 2 r g^{\text {tmlWjl }} /$ SzJ (NSG) mice were transplanted with LOUCY cells transduced with scrambled and RUNX2-specific shRNAs. We observed prolonged survival of animals inoculated with RUNX2-depleted cells compared with control mice (Figure 3J). In addition, postnecropsy analyses of the animals sacrificed 41 days after injection revealed decreased levels of leukemic cells infiltrating the BM, spleen, and meninges (Figure 3K). We also tested to determine whether KMT2A-R leukemia is dependent on RUNX2 in vivo. KMT2A-MLLT1 PDX cells were transduced with RUNX2 shRNA containing a GFP selection marker and injected into recipient NSG mice. While leukemia engraftment was evident in control mice, GFP-positive cells were absent or significantly reduced in the peripheral blood, BM, and spleen (Figure $3 \mathrm{~L}$ ) in animals inoculated with RUNX2-depleted cells. Together, these results suggest that RUNX2 is a dependency factor for survival of immature and KMT2A-R T-ALL.

RUNX2 directly regulates an oncogenic transcription signature in immature and KMT2A-rearranged T-ALL, and its expression is regulated by KMT2A fusion proteins. To identify the genes that are directly regulated by RUNX2 in T-ALL, we performed ChIPSeq on KMT2A-R KARPAS-45 and immature/ETP PER-117 cells. MACS2 peak calling resulted in 42,948 and 21,027 peaks, respectively. Motif analyses revealed that the canonical RUNX-binding motif was most significantly enriched in both cell lines (Figure 4, A and B, and Supplemental Table 1). Other highly enriched motifs in the RUNX2-bound regions included the ETS motif in KARPAS45 and the ETS and GATA motifs in PER-117 (Figure 4, A and B), suggesting that RUNX2 acts in concert with other hematopoietic transcriptional regulators, as previously reported for RUNX1 in the context of hematopoietic development and T-ALL $(43,44)$. Furthermore, public ChIP data sets showed that RUNX2-bound regions in KARPAS-45 overlap with binding sites occupied by RUNX1 and ETS1 in JURKAT cells. Similarly, RUNX2-binding sites in PER-117 coincided with RUNX1, ETS1, and GATA3 occupancy in JURKAT (Supplemental Figure 4, A and B). On a genome-wide level, binding of RUNX2 in KARPAS-45 and PER-117 was mainly found in promoter or first intron regions, while approximately $17 \%$ of RUNX2 occupancy was intergenic (Figure 4, C and D).

To further elucidate the transcriptional program regulated by RUNX2, we performed RNA-Seq upon shRNA-mediated RUNX2 knockdown in KARPAS-45. We found 169 genes significantly $\left(P_{A d j}\right.$ $<0.05$ ) downregulated and 372 genes upregulated 48 hours upon knockdown, while 768 and 1099 genes were down- and upregulated after 72 hours, respectively. This further supports the notion 
A
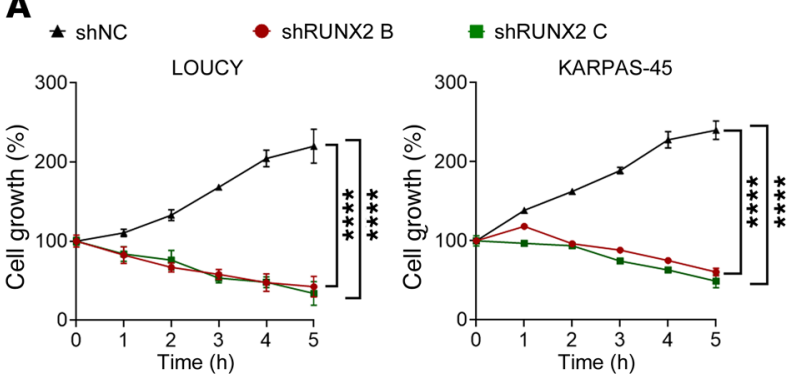

C

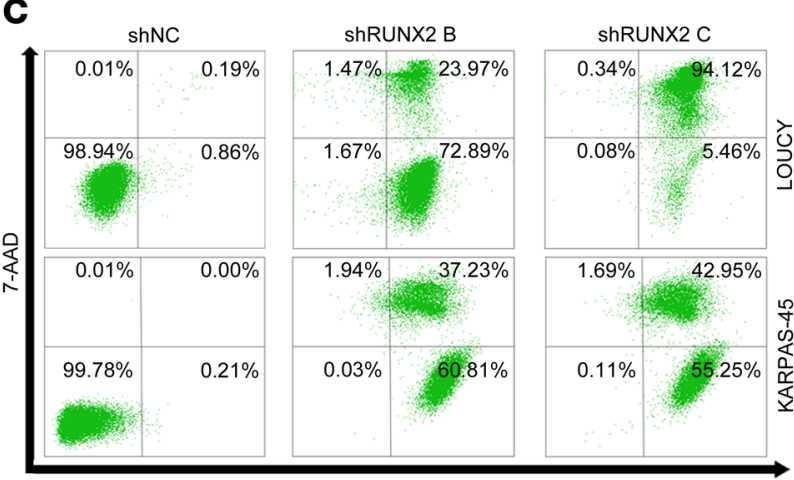

E

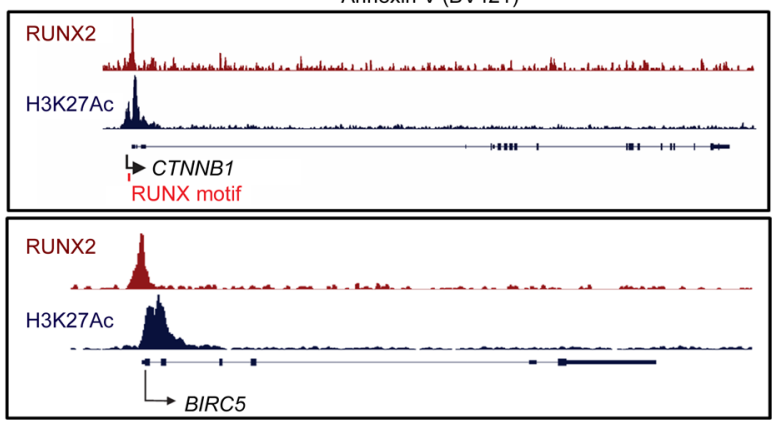

$\mathbf{F}$

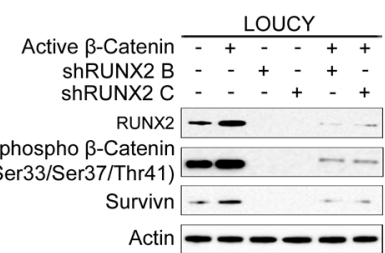

G

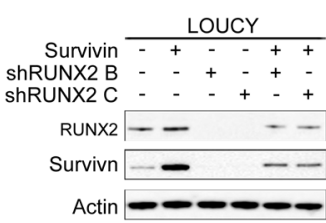

$\mathbf{K}$
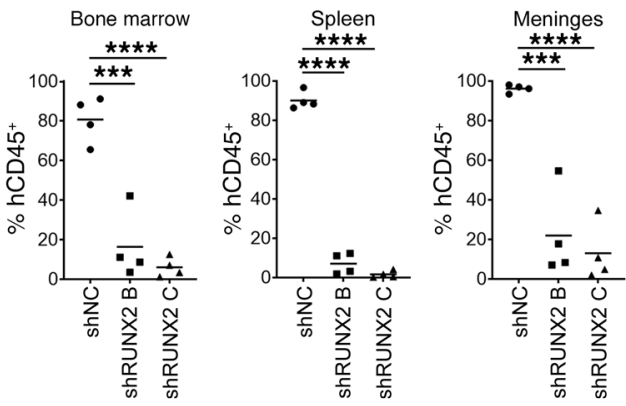

H

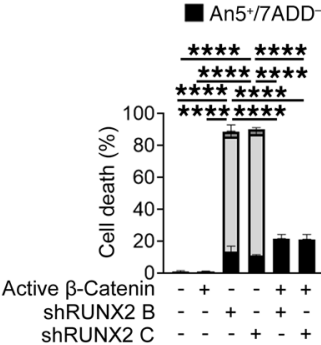

$\mathbf{L}$
B
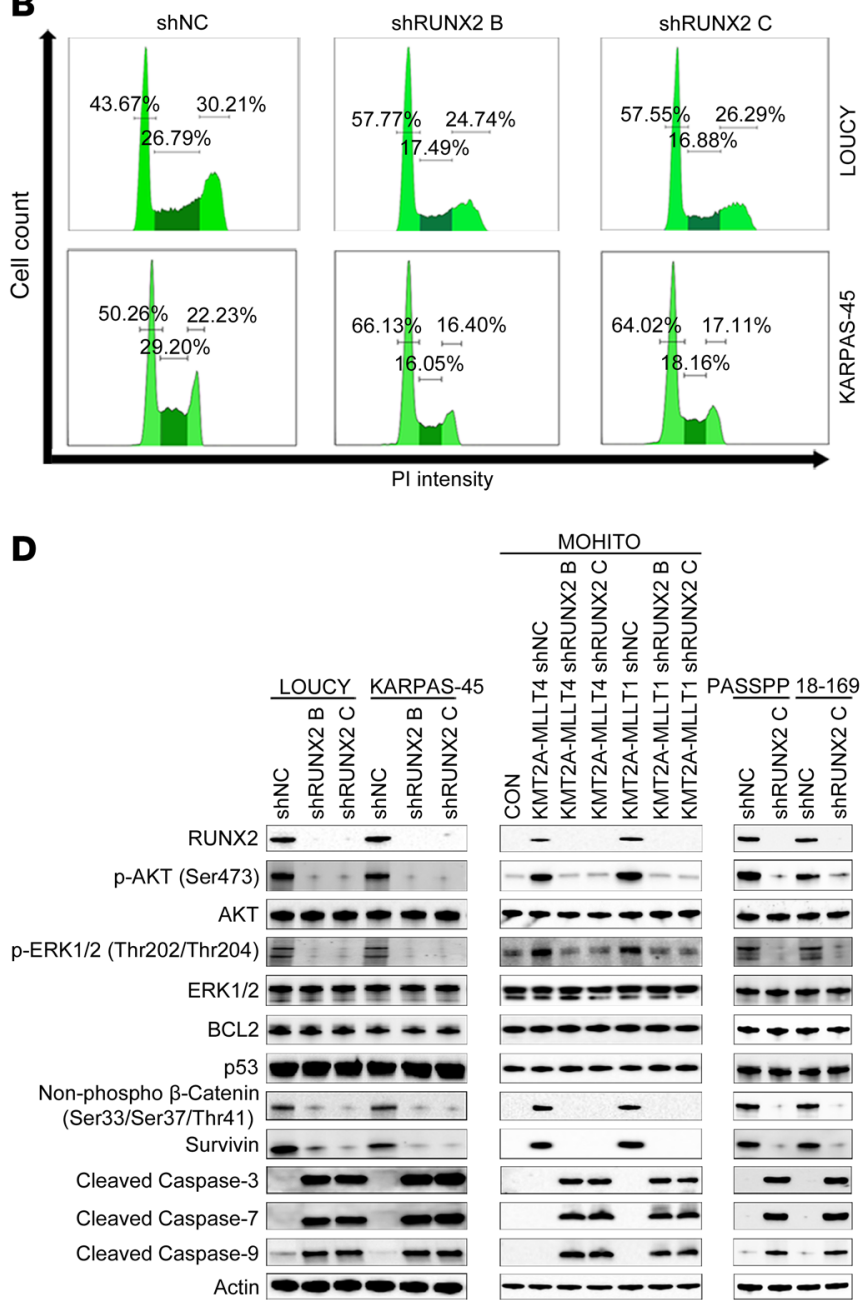

I

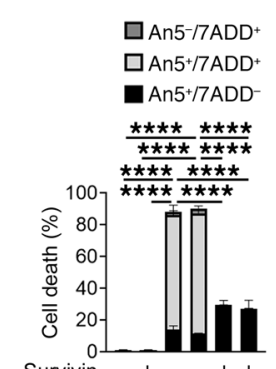

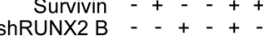
$\begin{array}{lll}\text { ShRUNX2 } & \cdots+\ldots+ \\ \text { shRUNX2 C } & \cdots & +\end{array}$

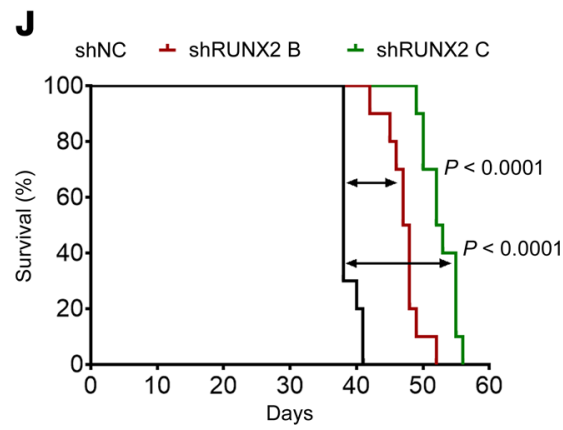

D

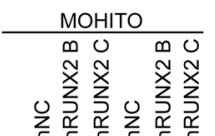

t5

- -
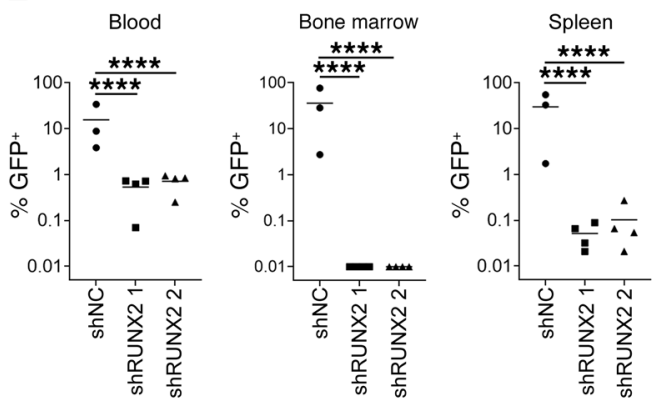
Figure 3. RUNX2 regulates T-ALL survival. (A) Cell growth of LOUCY and KARPAS-45 cells transduced with RUNX2 shRNAs (shRUNX2 B, shRUNX2 C) and scrambled control (shNC). Data are shown as mean \pm SD for 1 of 3 independent experiments performed in triplicate. (B) Flow cytometry cell-cycle analysis using propidium iodide staining (48 hours). (C) Apoptotic cell death in T-ALL cells stained with annexin V/7-AAD ( 96 hours). (B and C) Data are representative of 1 of 3 independent experiments. (D) Human LOUCY and KARPAS-45 cells, murine MOHITO cells expressing control plasmid (CON), KMT2A-R (KMT2A-MLLT1, KMT2A-MLLT4), and primary T-ALL cells (PASSPP, 18-169) were transduced with RUNX2 shRNAs and scrambled control. Western blot analysis of the indicated proteins. (E) RUNX2 and H3K27ac ChIP-Seq at the CTNNB1 ( $\beta$-catenin) and BIRC5 (survivin) loci in KARPAS-45. Forced expression of (F) active $\beta$-catenin and (G) survivin in LOUCY with or without shRNA-mediated RUNX2 depletion. (H and I) Annexin V/7-ADD staining in LOUCY ( 96 hours). Data are shown as mean \pm SD for 3 independent experiments. AnV, annexin V. (J) Kaplan-Meier survival curve analyses of NSG mice ( $n=10$ /group) transplanted with $10^{6}$ LOUCY cells expressing shRUNX2 B, shRUNX2 C, or scrambled control (median log-rank Mantel-Cox test). (K) Flow cytometric quantification of human CD45+ cells in mice ( $n=4 /$ group) euthanized 41 days after inoculation with $10^{6}$ transduced LOUCY cells. (L) The levels of GFP' ${ }^{+}$cells were determined in blood (day 30), BM, and spleen (day 100) in animals injected with PDX samples harboring KMT2A-MLLT-1. (A) Repeated measure ANOVA with Tukey's multiple comparisons test; ( $\mathbf{H}, \mathbf{I}, \mathbf{K}$, and $\mathbf{L}$ ) 1-way ANOVA with Tukey's multiple comparison test. ${ }^{* *} P<0.0005$; ${ }^{* * *} P<0.0001$. (D, $\mathbf{F}$, and $\mathbf{G}$ ) Representative blots from at least 2 separate experiments.

that RUNX2 not only acts as a transcriptional activator, but also as a repressor. Functionally, genes downregulated by RUNX2 knockdown showed enrichment for gene sets related to the cell cycle, DNA damage, and metabolism (Supplemental Table 2). Notably, CXCR4 was one of the most highly expressed genes that was significantly downregulated. MYC and CDK4 were also downregulated, while DICER1 was upregulated, among others (Figure $4 \mathrm{E}$ and Supplemental Table 2). Integration of ChIP and RNA-Seq data in KARPAS-45 showed an increase in differentially regulated genes when RUNX2 was bound on the promoter (Supplemental Figure 4C and Supplemental Table 3).

To analyze the functional significance of RUNX2 binding, we used the Genomic Regions Enrichment of Annotations Tool (GREAT) (45) to associate genes to RUNX2-bound regions followed by enrichment analysis (Supplemental Table 1). Both KARPAS-45 and PER-117 showed enrichment for T cell signaling and proliferation upon analysis of Gene Ontology (GO) biological processes. Cell-adhesion genes were also enriched in KARPAS-45 (Figure $4 \mathrm{~F}$ ), while histone-modification genes were enriched in PER-117 (Figure 4G). CXCR4 signaling was the only MSigDB pathway in KARPAS-45 for which enrichment was found (Supplemental Figure 4D). Enrichment of T cell leukemia was found upon analysis of disease ontology in PER-117 and revealed that RUNX2 is bound on regulatory regions of archetypical leukemia driver genes, such as NOTCH1, MYB, and LMO2 (Supplemental Table 1), and also on the distal MYC enhancer N-Me (ref. 46 and Supplemental Figure 4E). Importantly, in KARPAS-45, MSigDB perturbations showed that RUNX2-regulated genes were enriched for KMT2A-R signature genes (Figure $4 \mathrm{H}$ ). Furthermore, there was clear binding of RUNX2 on the KMT2A promoter in KARPAS-45 (Figure 4I). We next sought to determine whether, in addition to coregulation of the KMT2A-R gene expression signature, RUNX2 might be directly involved in driving expression of the KMT2A fusion protein itself, establish- ing a regulatory feed-forward loop. To test this hypothesis, we performed ChiP-qPCR on KARPAS-45 and 2 primary KMT2A-R T-ALL samples. Significant enrichment for KMT2A and RUNX2 promoter regions was found in RUNX2 and N-terminal KMT2A (KMT2A ${ }^{\mathrm{N}}$ ) immunoprecipitates, respectively (Figure 4J). Furthermore, a luciferase reporter assay using the KMT2A promoter showed that RUNX2 silencing significantly decreased $K M T 2 A$ promoter activity (Figure 4K). Comparatively, loss of the RUNX2-binding site abrogated KMT2A promoter activity, suggesting that RUNX2 is a core controller of the KMT2A promoter. Moreover, shRNA-mediated inactivation of RUNX2 resulted in decreased levels of KMT2A ${ }^{\mathrm{N}}$ in primary KMT2A-R T-ALL samples (Figure 4L).

Others have previously reported RUNX2 upregulation in KMT2A-R leukemia originating from B lymphoid or myeloid lineages $(47,48)$. Using published microarray data sets, we confirmed elevated RUNX2 expression levels in both precursor B cell acute lymphoblastic leukemia (49) and acute myeloid leukemia with KMT2A-R (ref. 50 and Supplemental Figure 4, F and G). To investigate whether RUNX2 is directly upregulated by KMT2A fusion proteins, we transduced murine MOHITO and $\mathrm{Ba} / \mathrm{F} 3$ cells with KMT2A-MLLT4 and KMT2A-MLLT1 fusions that are prevalent in $K M T 2 A-\mathrm{R}$ T-ALL $(8,16)$. The expression of $K M T 2 A-R$ resulted in elevated RUNX2 mRNA and protein (Supplemental Figure $4 \mathrm{H}$ ). We next created KMT2A-MLLT1-driven leukemic cells by transduction of mouse BM progenitors, followed by serial replating. Differential gene-expression analysis of KMT2A-MLLT1-transformed cells versus the progenitor cells revealed a highly specific KMT2A expression signature characterized by increased expression of the Hoxa cluster genes and Meis1 (Supplemental Figure 4I and refs. 3, 13, 51). We observed significant upregulation of Runx2 in the transformed cells, and its expression gradually increased with each replating step (Figure 4, M and N). Public ChIP-Seq data analyses further confirmed binding of KMT2A fusion proteins on the RUNX2 promoter in human (52) and mouse (ref. 53 and Supplemental Figure 4J). Interestingly, cross-comparison of several ChIP-Seq data sets revealed that RUNX2 belongs to a small core set of genes that are directly bound by the 3 most common KMT2A fusion proteins (KMT2A-AFF1, KMT2A-MLLT3, KMT2A-MLLT1; Supplemental Figure $4 \mathrm{~K}$ and refs. 53-55). Collectively, these results suggest functional crosstalk between RUNX2 and KMT2A chimeras and that RUNX2 is a critical mediator of the KMT2A-Rdriven oncogenic program.

RUNX2 promotes T-ALL cell migration and adhesion in vitro. Since our ChIP-Seq and RNA-Seq data pointed to a role of RUNX2 in CXCR4 signaling, we further examined the effects of forced expression of RUNX2 using PF382 and CCRF-CEM cell lines, which had lower and/or undetectable RUNX2-expression levels (Figure 1, D and E). While RUNX2 upregulation did not alter cell growth, cell cycle, or apoptosis (Figure 5A and Supplemental Figure 5, A-C), it increased the expression of the CXCR4 receptor (Figure 5B and Supplemental Figure 5D) and enhanced T-ALL cell migration to the CXCL12 chemokine (Figure 5, C and D). Our ChIP-Seq confirmed RUNX2 binding on the promoter and enhancer of the CXCR4 gene (Figure 5E). RUNX2 upregulation increased the expression of critical regulators of cell contractility and motility, such as vinculin, paxillin, focal adhesion kinase (FAK), and ezrin-radixin-moiesin (ERM) (Figure 5F). Addi- 
A

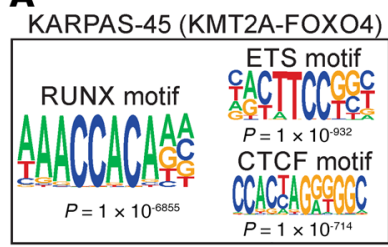

E

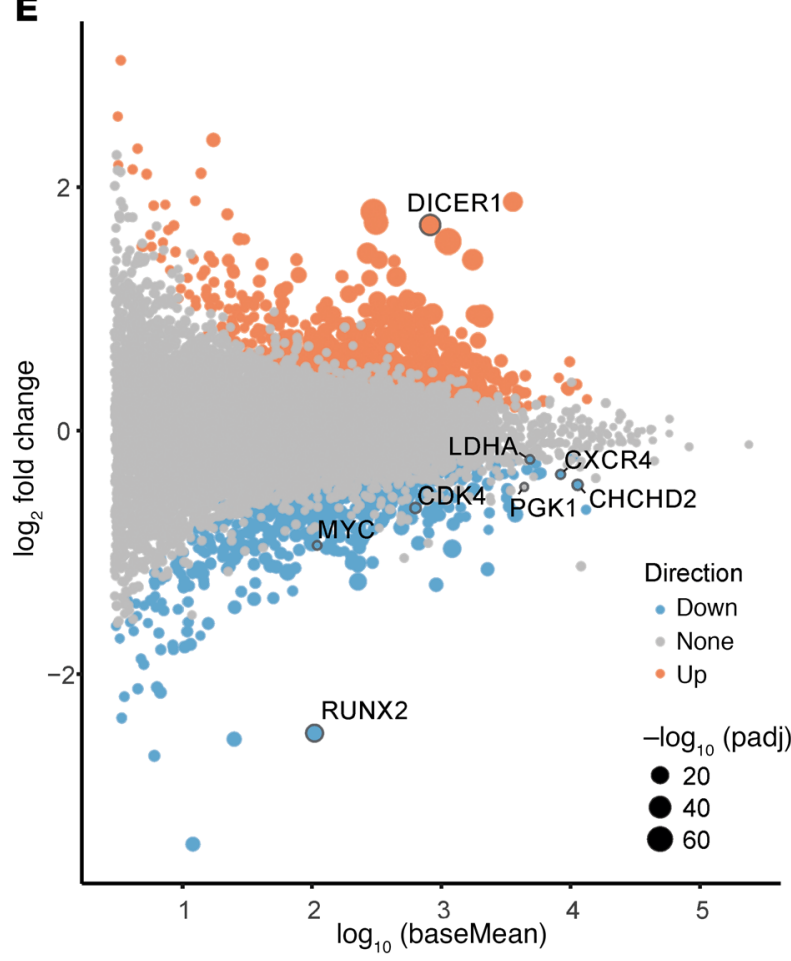

H

Enriched MSigDB perturbations - Karpas-45 Bound by FOXP3 Bound by PML-RARA in APL T lymphocyte differentiation stages Up in KMT2Ar pediatric AML (MLL signature 2) Up in KMT2Ar pediatric AML (MLL signature 1) Upregulated in Daudi cells expressing CD5

Top 100 up in KMT2Ar T-ALL (MLL-ENL) Down in...progenitors by RUNX1-RUNX1T1 16 q24 amplicon in breast cancer

Upregulated in rejected kidney transplant

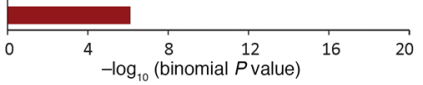

I

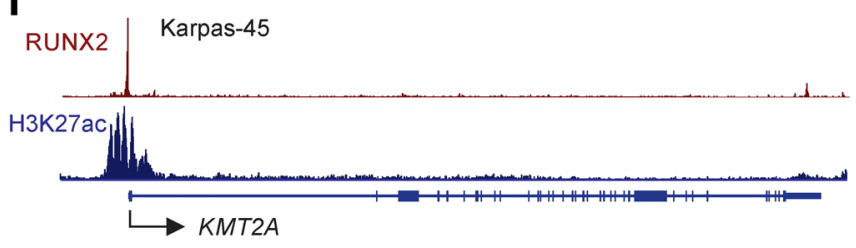

$\mathbf{L}$

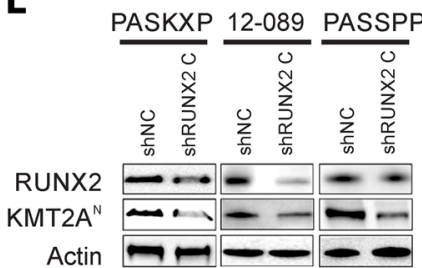

B

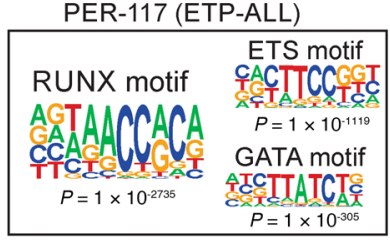

C

KARPAS-45

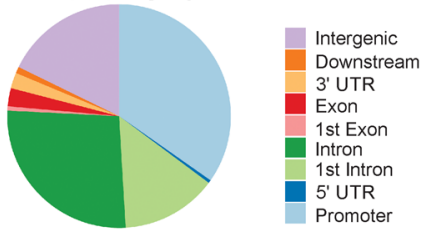

D

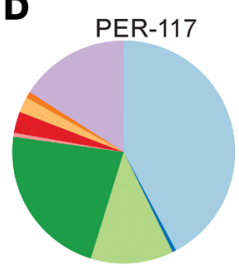

$\mathbf{F}$

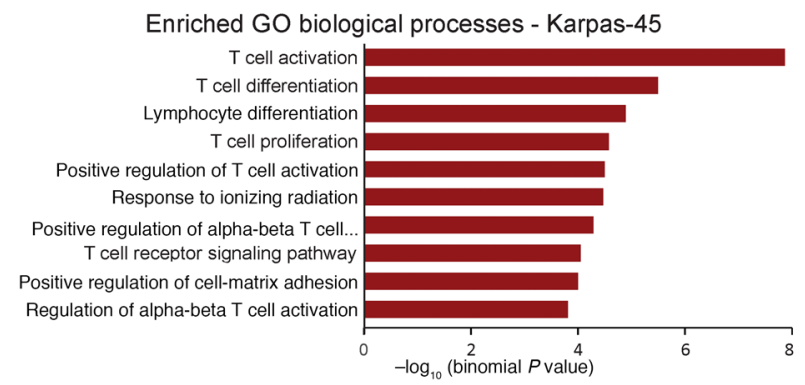

G

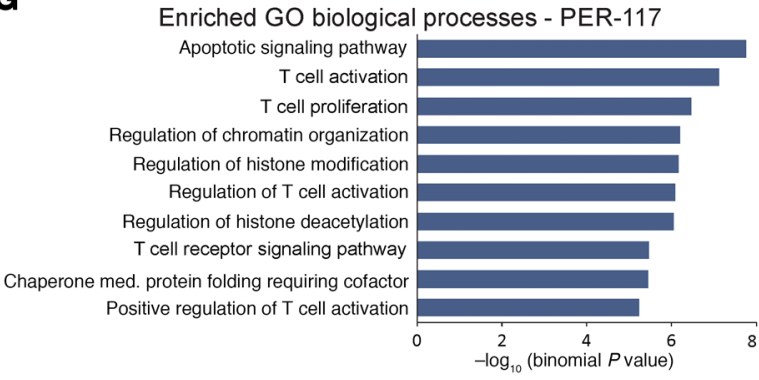

J

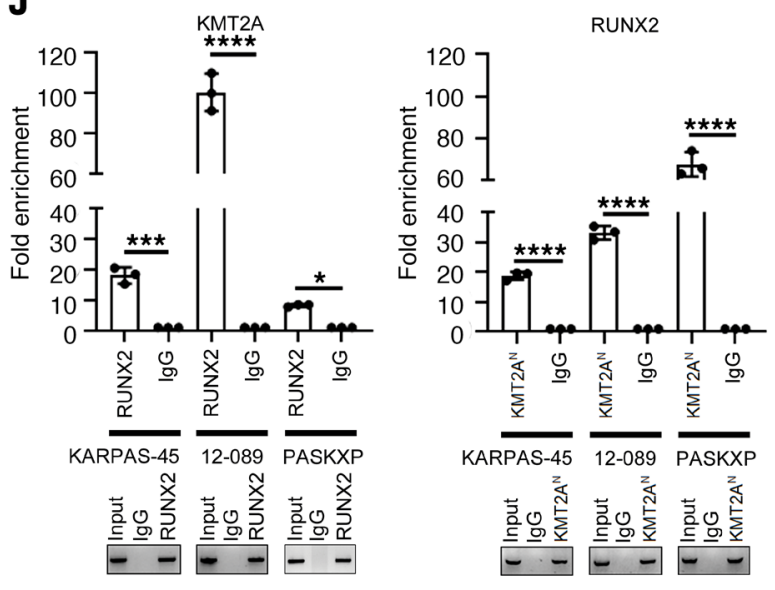

\section{K}

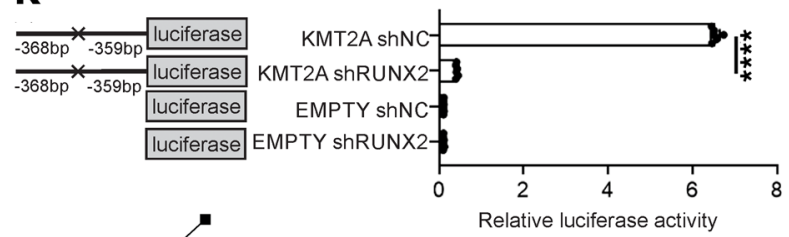

M

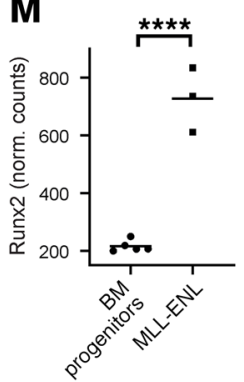

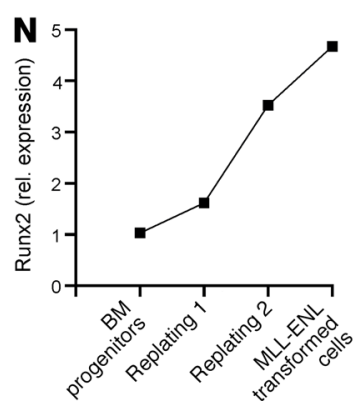


Figure 4. RUNX2 genome-wide binding profiles reveal a regulatory feedback loop with KMT2A fusion proteins. (A and B) Most highly enriched binding motifs according to HOMER upon RUNX2 ChIP-Seq in KARPAS-45 (KMT2A-R) and PER-117 (ETP). (C and D) Distribution of RUNX2 binding over different genomic regions in KARPAS-45 and PER-117. (E) MA plot showing differentially regulated genes $\left(P_{\text {Adj }}<0.05\right)$ in KARPAS- 45,72 hours after shRNA-mediated RUNX2 silencing vs. control in 5 biological replicates. (F-H) Top 10 enrichments of RUNX2-binding peaks based on functional annotation provided by GREAT (3.0.0). (I) RUNX2 and H3K27ac binding profile on the KMT2A gene region in KARPAS-45. (J) ChiP-qPCR on KARPAS- 45 cells and 2 KMT2A-R primary T-ALL patient samples. Enrichment of RUNX2 on KMT2A promoter (left); enrichment of N-terminus of KMT2A (KMT2A $)$ on RUNX2 promoter (right). (K) Luciferase reporter assay for KMT2A on HEK293 cells transduced with RUNX2 shRNA. The RUNX2 binding site is indicated as X ( -359 to -368 bp upstream of KMT2A coding starting site). (L) Silencing of RUNX2 leads to decreased levels of KMT2A $A^{\mathrm{N}}$ in primary T-ALL samples. (M) Runx2 expression in KMT2A-MLLT1 transformed cells compared with lineage-depleted mouse progenitor cells. (N) Gradually increased expression of Runx2 measured by qRT-PCR during KMT2A-MLLT1 driven transformation over different replatings. (J) Unpaired 2-tailed $t$ test with Holm-Šidák correction for multiple comparisons; (M) unpaired 2-tailed $t$ test. ${ }^{*} P<0.05 ;{ }^{* *} P<0.0005$; ${ }^{* * *} P<0.0001$.

tionally, RUNX2 enhanced the expression of proteins linked to epithelial-mesenchymal transition (EMT), further supporting its role in promoting T-ALL cell migration and adhesion (Figure 5F). KMT2A-R leukemias commonly express EMT genes $(14,56)$. We found that KMT2A-MLLT1 and KMT2A-MLLT4 induce the expression of EMT regulators (Snail, ZEB1, and vimentin) in MOHITO and $\mathrm{Ba} / \mathrm{F} 3$ cells; this was reduced by RUNX2 depletion (Figure $5 \mathrm{~F}$ and Supplemental Figure 5E). shRNA-mediated RUNX2 knockdown in LOUCY, KARPAS- 45 , and 2 primary T-ALL samples harboring KMT2A-R further supported the role of RUNX2 in positive regulation of EMT genes (Figure 5F and Supplemental Figure 5E). CXCR4-CXCL12 signaling mediates integrin activation, leading to an increased adhesion of leukemic cells to extracellular matrix, including fibronectin (57). RUNX2 upregulation enhanced the adhesion of T-ALL cells to fibronectin and increased the expression of VLA-4 in the tested cells (Figure 5, G and H). Collectively, these results suggest that RUNX2 positively regulates T-ALL cell chemotaxis, migration, and adhesion.

RUNX2 exacerbates T-ALL progression and extramedullary infiltration. Given that RUNX2 enhanced leukemic cell migration and adhesion in vitro, we subsequently performed in vivo experiments to study the effects of RUNX2 upregulation on T-ALL progression and medullary/extramedullary infiltration. Strikingly, forced expression of RUNX2 accelerated disease progression in mice engrafted with PF382 RUNX2 overexpressing (RUNX2 OE) cells compared with control animals (Figure 5I), with median survival decreasing in the RUNX2 OE group by $38 \%(P<0.0001)$. Tissue analysis of RUNX2 OE mice sacrificed 34 days after intrafemoral transplantation revealed profound evidence of disease dissemination in the BM and extramedullary infiltration of the liver, spleen, and meninges relative to mice in the control group (Figure 5, J and $\mathrm{K}$, and Supplemental Figure 5, F and G).

Given the role of CXCR4/CXCL12 in T-ALL homing and maintenance (58-60), we studied how RUNX2 contributes to homing of T-ALL cells in the BM using a noncompetitive and competitive approach. Total BM was collected 24 hours after injection of NSG mice with RUNX2 OE and negative control cells. In both experiments, a significantly higher number of RUNX2 OE cells were recovered from the femurs compared with negative control cells (Figure 5L). Together, these results suggest that RUNX2 potentiates BM homing of T-ALL cells and disease progression.

To further characterize how RUNX2 exacerbates disease progression in vivo, we evaluated the levels of human and mouse cytokines in blood serum of engrafted NSG mice. Mice injected with RUNX2 OE cells presented with higher levels of human TNF- $\alpha$ and IL-10 in blood serum compared with controls 28 days after inoculation (Figure 5M). Additionally, mouse cytokine profiling revealed elevated levels of several chemokines, such as CXCL10, CCL2, CCL4, and CCL5, as well as IL-6 cytokine, while the levels of IL-1 $\alpha$ were decreased (Supplemental Figure 5H). While these observations may suggest a role of RUNX2 in regulating cytokine signaling and host immune response, further studies are necessary to elucidate the mechanism underlying RUNX2 contribution to T-ALL engraftment and dissemination.

RUNX2 increases metabolic activity of T-ALL cells. Given that cell migration is an energy-demanding process, we subsequently studied the effects of RUNX2 on T-ALL metabolism. We first assessed how RUNX2 regulates glycolysis, a common alternative energy source in leukemia. Forced expression of RUNX2 resulted in increased extracellular acidification rate (ECAR), glycolytic flux, glucose uptake, and expression of the glucose transporter GLUT1 (Figure 6, A and B, and Supplemental Figure 6, A-C). Treatment of transduced T-ALL cell lines with a glucose analog, 2-deoxy-Dglucose (2DG), reduced T-ALL cell migration to CXCL12 (Figure 6C). Furthermore, we found that KMT2A-R increased glycolytic capacity, glucose uptake, and GLUT1 levels in MOHITO and Ba/ F3 cells and that RUNX2 depletion reduced these processes in MOHITO, Ba/F3, and LOUCY cells (Figure 6, D and E, and Supplemental Figure 6, D-K). These results suggest that RUNX2 regulates glycolytic metabolism in KMT2A-R and immature leukemias. Strikingly, CCRF-CEM and PF382 cells overexpressing RUNX2 exhibited a higher oxygen consumption rate (OCR) and increased ATP production relative to control cells (Figure 6, F and G, and Supplemental Figure 6, L and M). The significant increase in both glycolytic and oxidative metabolism corresponded to increased OCR/ECAR ratios (Figure 6H), suggesting that RUNX2 enhances the metabolic activity of T-ALL cells. Similarly, an increase in OCR and ATP production was observed in MOHITO and Ba/F3 cells expressing KMT2A-R. Mitochondrial respiration was significantly reduced after genetic inactivation of RUNX2 expression in MOHITO and Ba/F3 with KMT2A-R or LOUCY cells (Figure 6I and Supplemental Figure 6, N-P). Expression of KMT2A-R in MOHITO and Ba/F3 cells increased OCR/ECAR ratios, suggesting an increase in metabolic potential. RUNX2 depletion reduced the OCR/ECAR ratios in LOUCY and KMT2A-R MOHITO and $K M T 2 A-\mathrm{R} \mathrm{Ba} / \mathrm{F} 3$ cells, suggesting its role in regulation of cell metabolism in immature and KMT2A-R leukemias (Figure 6J and Supplemental Figure 6, Q and R). RNA-Seq analyses showed that the metabolism-related genes $L D H A, C H C H D 2$, and PGK1 were highly expressed in KARPAS- 45 and downregulated upon RUNX2 silencing (Figure 4 and Supplemental Table 2). RUNX2 binding on the promoter regions of $L D H A, C H C H D 2$, and PGK1 and on an upstream enhancer of $L D H A$ further suggested that these 
A

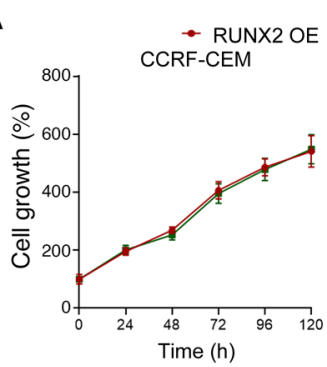

C

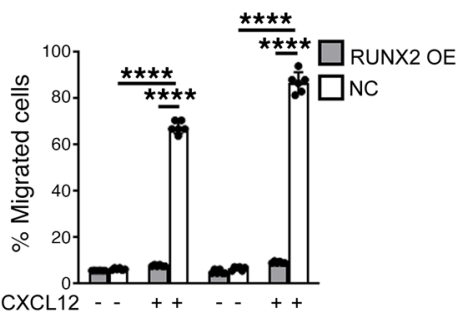

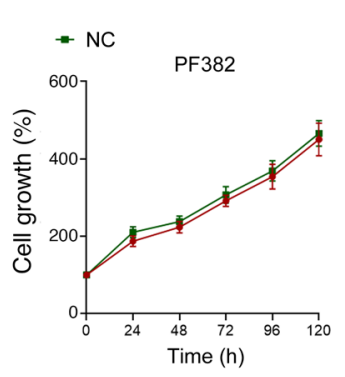

D

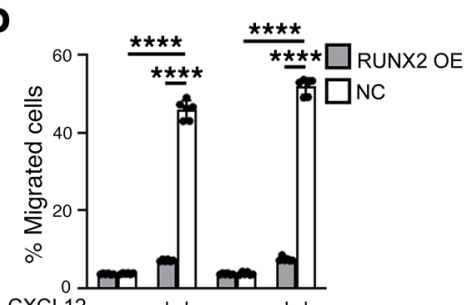

CXCL12 -
B

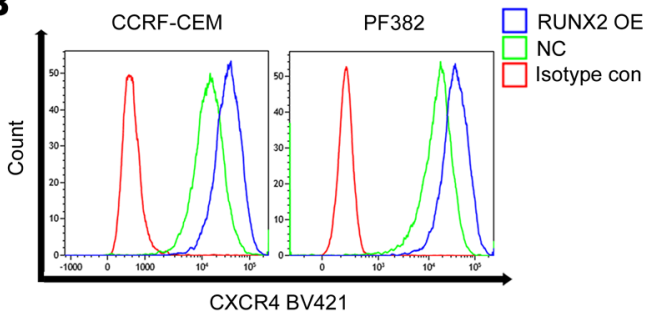

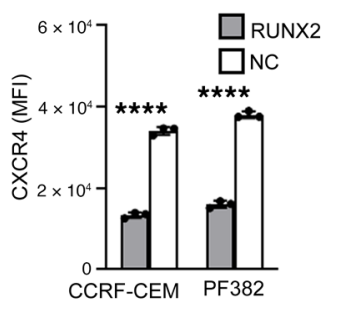

G
E

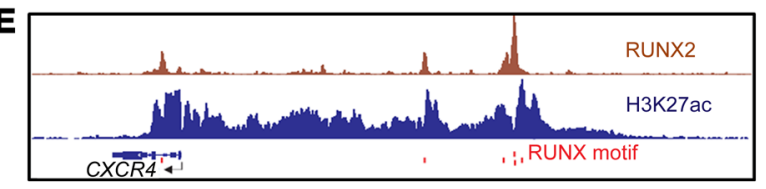

$\mathbf{F}$

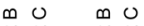

艾

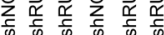

政

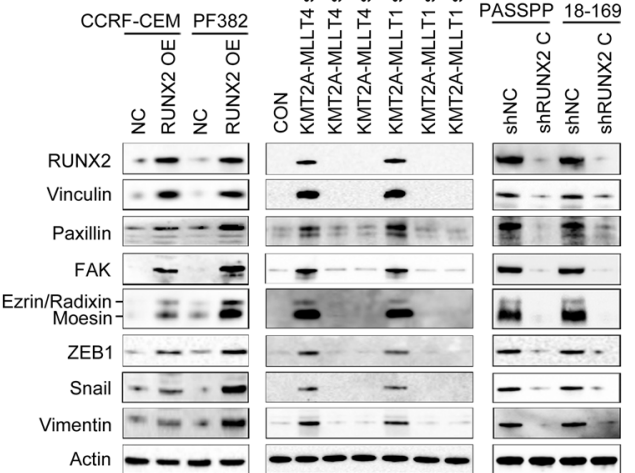

J
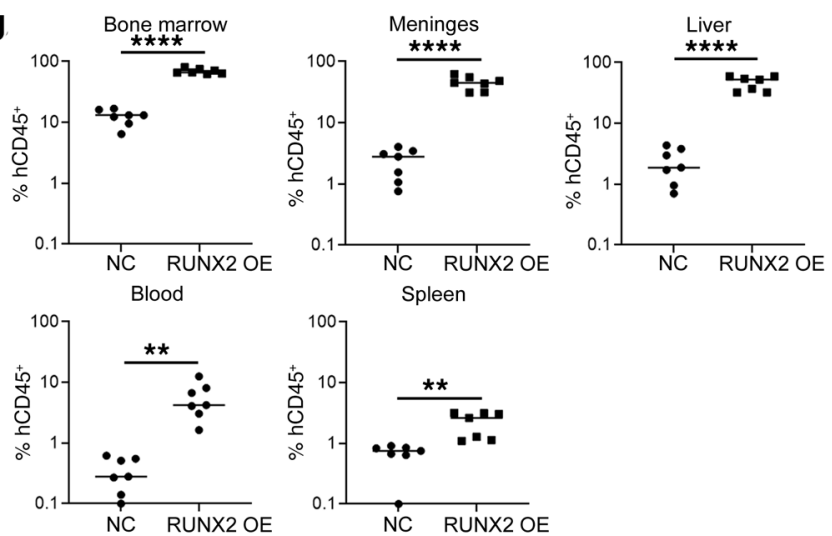

L
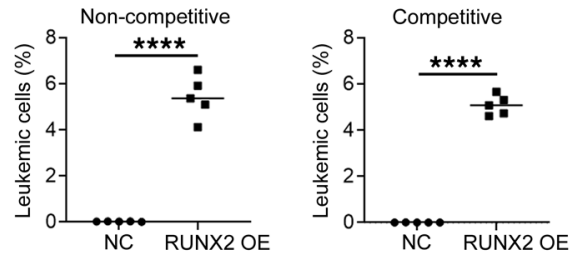

H

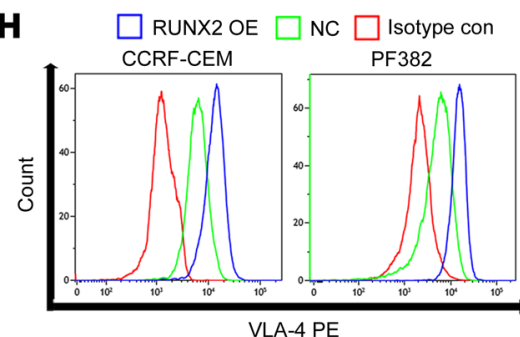

I

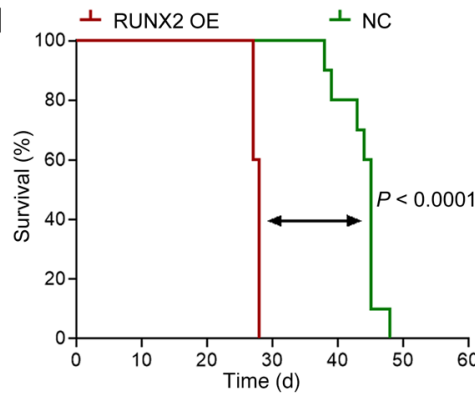

$\mathbf{K}$

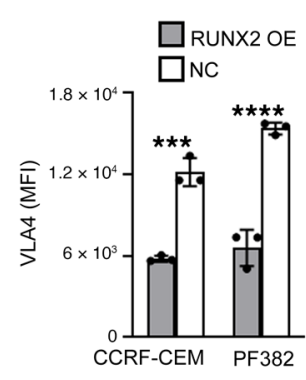

$\square$ RUNX2 OE $\square \mathrm{NC}$
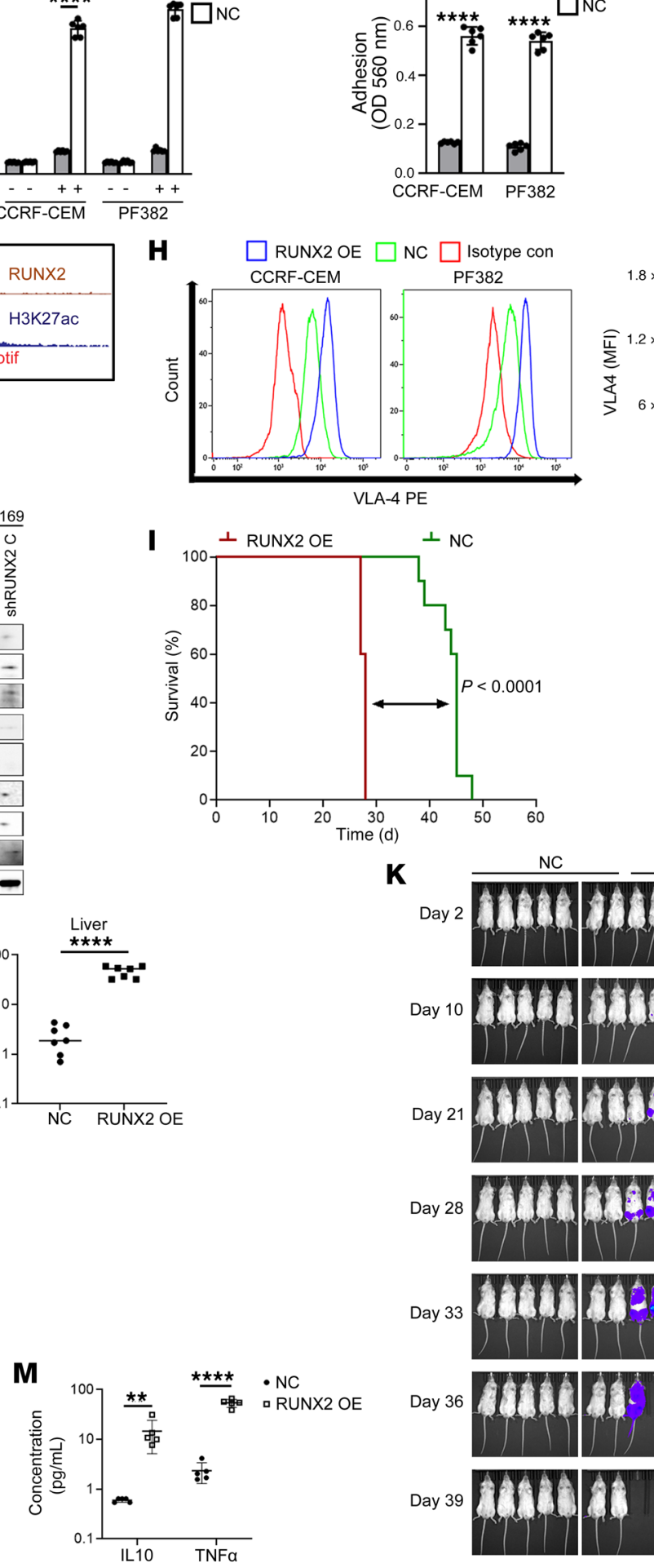
Figure 5. RUNX2 upregulation promotes T-ALL cell migration and leukemia progression. (A) Growth of CCRF-CEM and PF382 cells transduced with RUNX2-expressing (RUNX2 OE) or negative control (NC) plasmids. Data are shown as mean \pm SD, 1 of 3 independent experiments performed in triplicate, repeated measure ANOVA with Tukey's multiple comparisons test. (B) CXCR4 levels; representative histograms (left); median fluorescent intensity (MFI) $\pm S D, 3$ independent experiments (right). Leukemic cell migration (6 hours) through (C) $3 \mu \mathrm{m}$ porous membrane or (D) HUVEC monolayer \pm CXCL12 $(50 \mathrm{ng} / \mu \mathrm{l})$. Data are shown as mean \pm SEM, 3 separate experiments performed in duplicate. Two-way ANOVA with Tukey's multiple comparison correction. (E) RUNX2 and H3K27ac binding at the CXCR4 locus in KARPAS-45. (F) CCRF-CEM and PF382 transduced with RUNX2 OE or negative control; MOHITO cells expressing KMT2A-R (KMT2A-MLLT1, KMT2A-MLLT4) or negative control and primary T-ALL cells (PASSPP, 18-169) transduced with shRUNX2 B, shRUNX2 C, and scrambled control. Immunoblotting with indicated antibodies $(n=$ 2). (G) Adhesion to fibronectin (2 hours). Data are shown as mean $\pm S D, 3$ independent experiments performed in duplicate. (H) VLA-4 levels; representative histograms (left); MFI $\pm \mathrm{SD}, 3$ separate experiments (right). (I) Kaplan-Meier plot of NSG mice ( $n=10$ /group) injected i.v. with $10^{6} \mathrm{PF} 382$ cells (log-rank test). (J) Human CD45+ cells (day 34) and (K) bioluminescence imaging (NSG mice, $n=7 /$ group) after interfemoral implantation of PF382 cells $\left(3 \times 10^{5}\right)$. (L) BM homing of leukemic cells (24 hours); NSG mice ( $n=5$ /group) received i.v. PF382 RUNX2 OE and negative control cells (107; noncompetitive) or the mixture of fluorescently labeled PF382 RUNX2 OE (DsRed) and negative control (AmCyan) cells (1:1; 107; competitive). Unpaired and paired 2-tailed $t$ tests, respectively. (M) Human cytokines in blood serum of NSG mice inoculated with $10^{6}$ PF382 cells (day 28). (B, $\mathbf{G}, \mathbf{H}, \mathbf{J}$, and $\mathbf{M}$ ) Unpaired $t$ test with Holm-Šidák multiple comparisons correction. ${ }^{* *} P<0.005$; ${ }^{* *} P<0.0005$; ${ }^{* * *} P<0.0001$.

genes are direct transcriptional targets for RUNX2 (Figure 6K). RUNX2 overexpression in PF382 and CCRF-CEM cells resulted in increased expression of LDHA, CHCHD2, and PGK1 transcript and protein levels (Figure 6L and Supplemental Figure 6S). Conversely, RUNX2 inactivation in T-ALL cell lines and primary samples markedly reduced mRNA and protein levels of the tested metabolic regulators (Figure 6L and Supplemental Figure 6, T-V). Collectively, our results indicate that RUNX2 plays a role in the regulation of glycolytic and mitochondrial metabolism in T-ALL cells.

RUNX2 regulates mitochondrial dynamics and biogenesis. With evidence that RUNX2 regulates oxidative and glycolytic metabolism in T-ALL cells, we sought to understand its effect on mitochondrial dynamics. RUNX2 upregulation increased mitochondrial membrane potential and reduced ROS levels in the cell lines tested (Figure 7, A and B). Consistently, expression of KMT2A-R in MOHITO and Ba/F3 cells enhanced mitochondrial membrane potential and lowered ROS levels, and that effect was abrogated in RUNX2-depleted LOUCY cells (Figure 7, C and D, and Supplemental Figure 7, A-D). On a protein level, RUNX2 positively regulated mitochondrial fusion (OPA1, mitofusin 1, and mitofusin 2) and fission (P-Drp1, FIS1, and MFF) as well as proteins associated with mitochondrial biogenesis (PGC1 $\alpha$, NRF-1, TOMM20; Figure 7E). RUNX2 induced the activation of AMPK, which is known to regulate mitochondrial dynamics and biogenesis, and induced phosphorylation of major upstream kinases of AMPK, LKB1, and CAMKK2 (Figure 7F and ref. 61). The expression of mitochondrial dynamics and biogenesis mediators and AMPK pathway activation were also increased in MOHITO and $\mathrm{Ba} / \mathrm{F} 3$ cells expressing KMT2A-R (Figure 7, E and F, and Supplemental Figure 7, E and F). Conversely, depletion of RUNX2 in LOUCY, KARPAS-45, and
MOHITO or Ba/F3 with KMT2A-R decreased levels of the tested proteins and AMPK activation (Figure 7, E and F, and Supplemental Figure 7, E and F). Furthermore, inhibition of mitochondrial fission or AMPK activation by treatment with Mdivi-1, a Drp1 inhibitor, or dorsomorphin, an AMPK inhibitor, reduced the ability of T-ALL cells to migrate to CXCL12, suggesting a role for RUNX2 in mitochondrial homeostasis and biogenesis in facilitating T-ALL chemotaxis (Figure 7, G and H). Together, these results show that RUNX2 positively regulates mitochondrial dynamics and biogenesis.

Immature T-ALL and KMT2A rearranged leukemias are vulnerable to pharmacological targeting of the RUNX-CBF $\beta$ interaction. It has been suggested that RUNX proteins can exert redundant functions in leukemia (62). We found that Runx2-deficient BM progenitors transformed by KMT2A-MLLT1 showed significantly upregulated Runx1 levels compared with Runx2 WT cells (Figure $8 \mathrm{~A})$. Although oncogenic transformation by KMT2A-R still occurs in Runx2-deficient cells, typical KMT2A target genes, such as Hoxa10, Hoxa11, and Hoxb13, showed reduced expression in Runx2deficient KMT2A-R cells (Figure 8, B-D). Given that Runx2 knockdown results in compensatory upregulation of Runx 1 and the oncogenic role of Runx1 in T-ALL (63) and KMT2A-AFF1 rearranged B-ALL (64), we reasoned that pan-RUNX inhibition might be a valid therapeutic targeting strategy for KMT2A-R and immature T-ALL. Interestingly, a series of small molecules that inhibit RUNXCBF $\beta$ binding have been recently developed (65) and were shown to be efficacious against RUNX1-driven T-ALL (63). We tested the effect of the inhibitor AI-10-104 on leukemic cell survival in a panel of T-ALL cell lines and found that the cell lines KARPAS- 45 and PER-117 were equally sensitive to this compound, as previously reported for other RUNX1-dependent T-ALL cell lines (ref. 63 and Figure $8 \mathrm{E}$ ). RNA-Seq was performed on KARPAS- 45 cells treated with $20 \mu \mathrm{M} \mathrm{AI}-10-104$ for 24 hours and compared with controls. Interestingly, gene sets related to mitochondrial membrane and metabolism were highly enriched in genes that were significantly downregulated by AI-10-104 treatment (Figure 8F and Supplemental Table 4), further supporting our findings that RUNX2 controls mitochondrial metabolism and dynamics in T-ALL. Using the most significantly down- and upregulated genes by AI-10-104 treatment as gene sets, we performed gene set enrichment analysis on the expression data of our RUNX2 shRNA knockdown experiment (Supplemental Figure 8). Highly specific enrichment of these sets in both the down- and upregulated genes upon shRNA knockdown suggests that AI-10-104 acts as a RUNX2 inhibitor in KARPAS-45. Finally, we examined the ex vivo sensitivity of PDX BM and spleen cells from 2 patients with KMT2A-MLLT1 or KMT2A-MLLT4 rearrangement toward AI-10-104 (Figure 8, G and H). These cells displayed $\mathrm{IC}_{50}$ values between 1.8 and $4.5 \mu \mathrm{M}$, which is within the same range as previously reported for primary T-ALL patient samples (63).

\section{Discussion}

Despite the emerging understanding of the role of RUNX2 in the aggressiveness and metastasis of solid tumors (30-33, 66-68), the molecular mechanisms underlying its oncogenic potential in leukemia remain largely unexplored. In this study, we identified RUNX2 as a critical regulator of cell growth and survival in highrisk T-ALL $(10,16)$ through regulation of tumor metabolism and leukemic cell migration. 
A

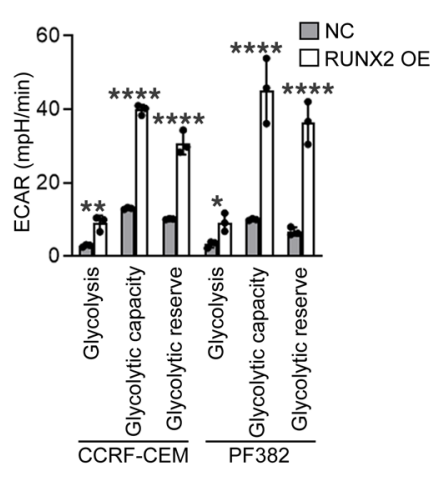

B $\square R U N \times 2$ OE $\square$ NC $\square$ lsotype con
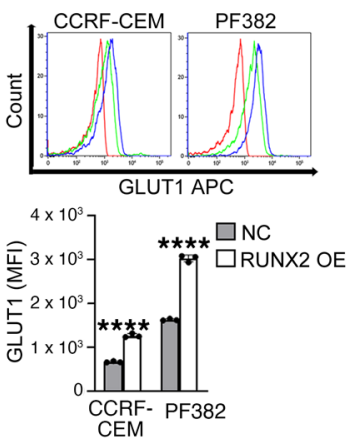

C

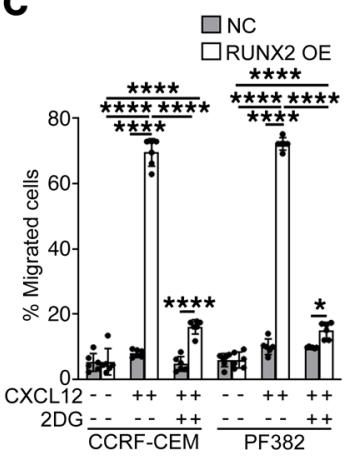

D $\square$ CON

D KMT2A-MLLT4 SANC $\square$ KMT2A-MLLT4 ShRUNX2B KMT2A-MLLT4 ShRUNX2 C KMT2A-MLLT1 ShNC

G KMT2A-MLLT1 ShRUNX2B

KMT2A-MLLT1 ShRUNX2 C

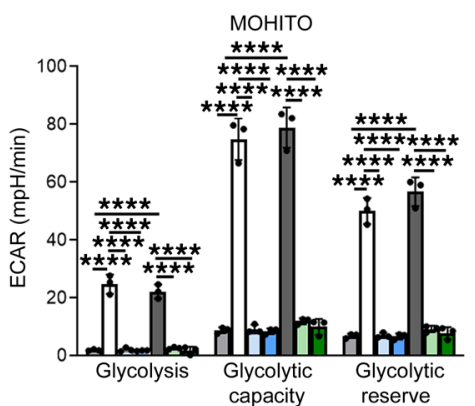

KMT2A-MLLT1 shNC KMT2A-MLLT1 ShRUNX2 B KMT2A-MLLT1shRUNX2 C $\square$ Isotype CON KMT2A-MLLT4 shRUNX2 B
KMT2A-MLLT4 shRUNX2 C $\square$ Isotype CON
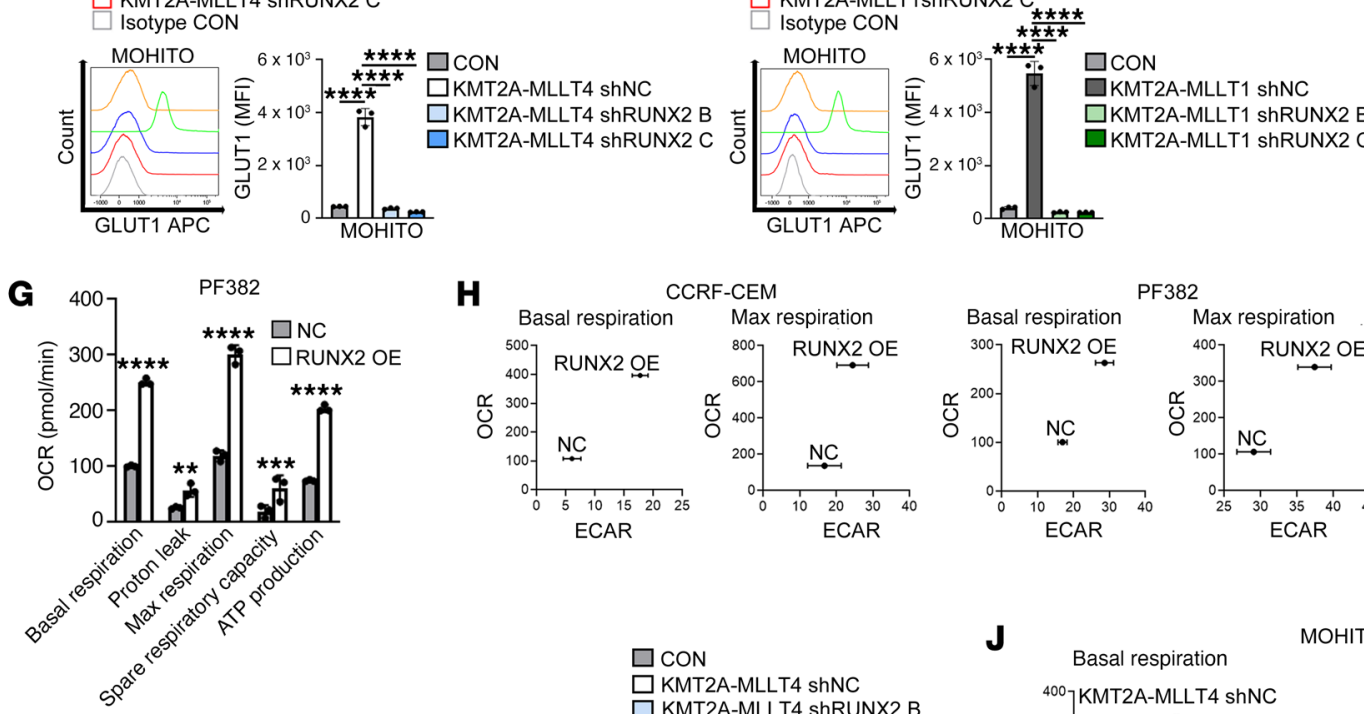

H

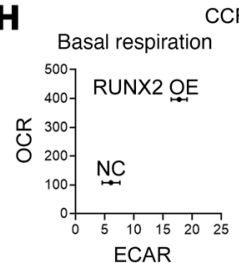

CCRF-CEM
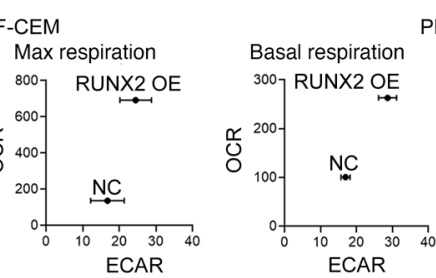

PF382

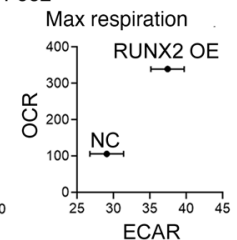

F $\square \mathrm{NC}$

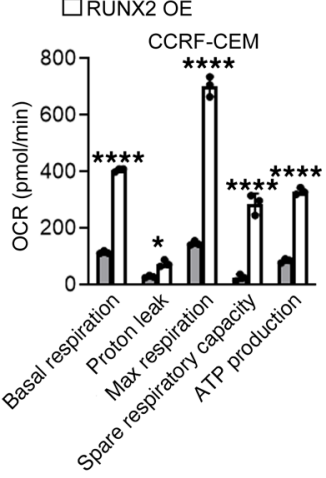

\section{I}

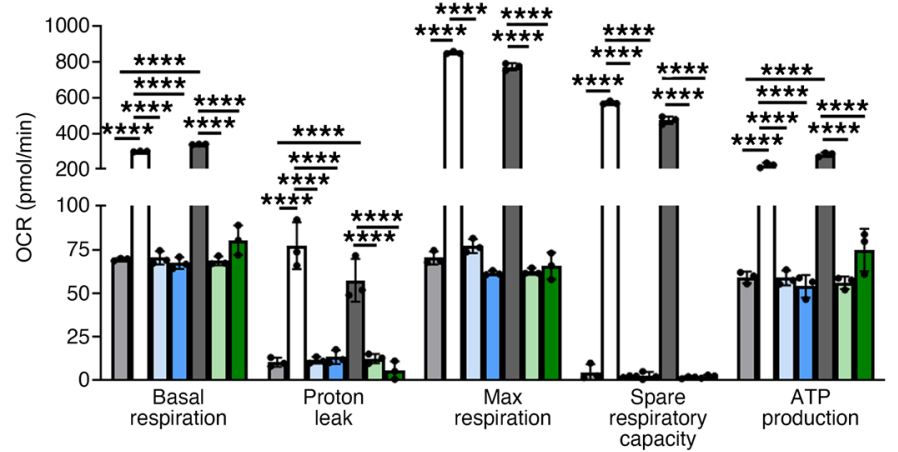

$\mathbf{K}$

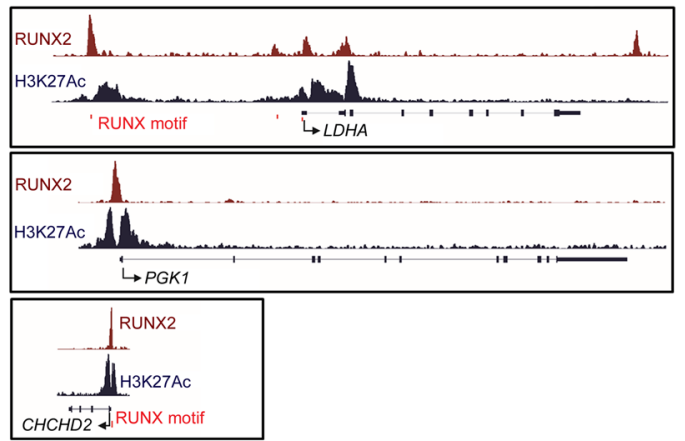
MOHITO $\square$ KMT2A-MLLT1 shNC

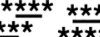

$\square$ CON

口 KMT2A-MLLT4 SSNC $\square$ KMT2A-MLLT4 ShRUNX2 B $\square$ KMT2A-MLLT4 ShRUNX2 C

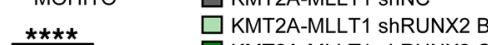
$\frac{\star \star \star \star \star}{\star \star \star \star \star ~} \square$ KMT2A-MLLT1 ShRUNX2 C
MOHITO
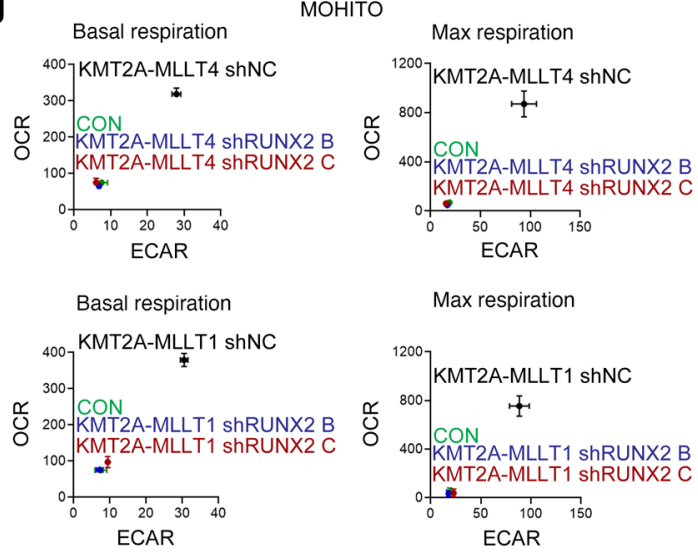

Max respiration

$\mathbf{L}$

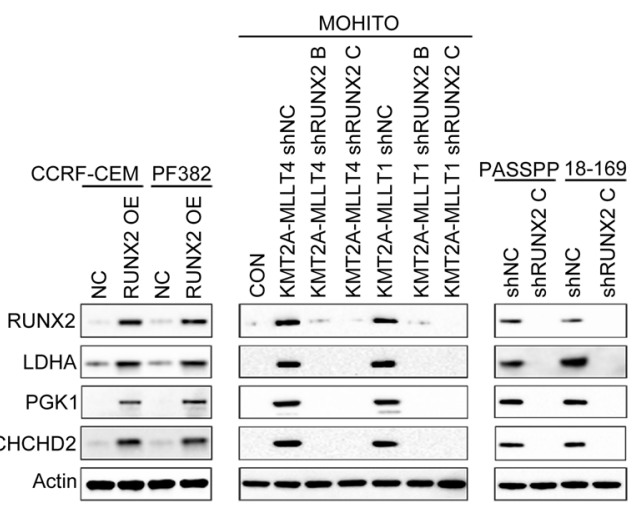

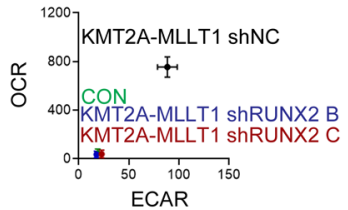

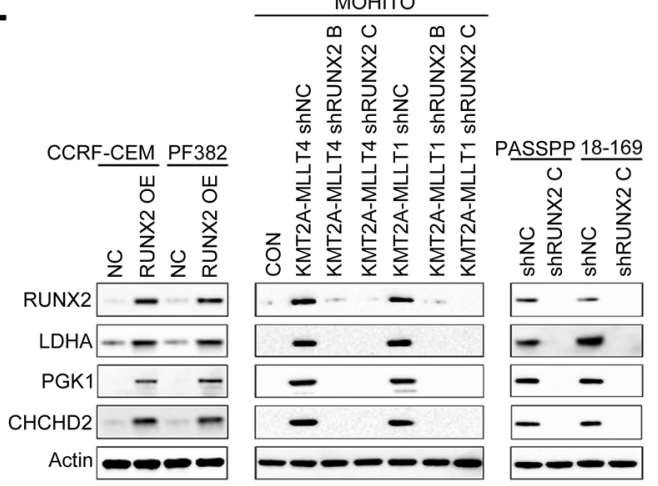


Figure 6. RUNX2 potentiates glycolytic and oxidative metabolism in T-ALL cells. (A) CCRF-CEM and PF382 cells were transduced with RUNX2 expressing (RUNX2 OE) and negative control plasmids. ECAR in glucose stress assay tested on the Seahorse XF24 Bioanalyzer. (B) Representative histograms for GLUT1 levels (top); MFI \pm SD, 3 separate experiments (bottom). (C) Migration of T-ALL cells pretreated with glucose inhibitor (2DC, 1 $\mathrm{mM} ; 16$ hours $) \pm \mathrm{CXCL} 12(50 \mathrm{ng} / \mu \mathrm{l} ; 6$ hours, $3 \mu \mathrm{m}$ porous membrane). Data are represented as mean $\pm S D$, 3 independent experiments performed in duplicate. (D) ECAR quantification and (E) GLUT1 levels in MOHITO-negative control cells, MOHITO-expressing KMT2A-R (KMT2A-MLLT4, KMT2AMLLT1) and transduced with RUNX2 shRNAs (shRUNX2 B, shRUNX2 C) or scrambled control. Representative histograms for GLUT1 expression (left); $\mathrm{MFI} \pm$ SD, 3 independent experiments (right). OCR upon RUNX2 forced expression in (F) CCRF-CEM and (G) PF382 cells analyzed using Mito Stress assay on the Seahorse XF24 Bioanalyzer. (H) Plot representing OCR/ECAR ratios in RUNX2 OE vs. control cells. (I) OCR and (J) ratio of OCR/ECAR for MOHITO control cells and MOHITO cells with or without KMT2A-R followed by shRNA-mediated RUNX2 silencing. (K) RUNX2 and H3K27ac binding on the LDHA, PGK1, and CHCHD2 gene regions in KARPAS-45. (L) Western blot analysis using indicated antibodies. Representative blots from at least 2 independent experiments. (A, D, and F-J) Data from 1 (mean \pm SD) of 2 independent experiments performed in triplicate. (A, B, F, and $\mathbf{G}$ ) Unpaired 2-tailed $t$ test with Holm-Šidák correction for multiple comparisons; (C, D, and I) 2-way ANOVA with Tukey's multiple comparison test; (E) 1-way ANOVA with Tukey's multiple comparison test. ${ }^{*} P<0.05$; ${ }^{* *} P<0.005$; ${ }^{* * *} P<0.0005 ;{ }^{* * *} P<0.0001$.

Our combined ChIP-Seq and RNA-Seq experiments identified RUNX2 as a transcription factor that directly and indirectly activates and represses a broad repertoire of genes in high-risk T-ALL with KMT2A-R or an immature/ETP immunophenotype. RUNX2-binding sites overlap with those of other hematopoietic transcriptional regulators, such as RUNX1, ETS1, and GATA3, suggesting interaction or possible redundant functions. For instance, similarly to observations for RUNX1 and RUNX3 (63), we found clear RUNX2 binding on the distal MYC enhancer N-Me (46) in KARPAS-45 and PER-117, coinciding with MYC downregulation upon RUNX2 depletion (Figure 4, Supplemental Figure 4, and Supplemental Table 1). Furthermore, enrichment analysis revealed that RUNX2 orchestrates important programs, such as cell cycle, metabolism, and $\mathrm{T}$ cell signaling, explaining its prosurvival role in T-ALL (Supplemental Tables 1 and 2). Furthermore, in KMT2A-R cells, we described RUNX2 binding and transcriptional regulation on the KMT2A promoter and, reciprocally, binding and regulation of the KMT2A fusion protein on RUNX2 (Figure 4). Together, these data provide compelling evidence for RUNX2 establishing a positive regulatory feed-forward loop with the KMT2A-fusion protein, thus supporting and reinforcing the KMT2A-R transcriptional signature. Consistently, we and others have identified RUNX2 within the most upregulated genes in $K M T 2 A-\mathrm{R}$ acute leukemias $(14,48,49)$. Integrative ChIP-Seq analyses demonstrated that RUNX2 belongs to a small set of genes directly bound by the 3 most common KMT2A fusion proteins (Supplemental Figure 4 and refs. 53-55). Further studies are warranted to define whether and how these genes contribute to the KMT2A-R oncogenic program.

Strikingly, we found that T-ALL cell survival depends on RUNX2 (Figure 3), while it did not seem critically required for normal hematopoiesis and $\mathrm{T}$ cell development (Figure 2). In T-ALL, RUNX2 inactivation leads to caspase-dependent apoptosis and delays disease progression in vivo. These findings are in line with previous reports highlighting the roles of RUNX2 in the development and survival of other hematological malignancies (27, 36, 37 ), in which RUNX2 cooperates with CBF $\beta$ and MYC in tumor development. Previous studies have linked RUNX2 to progression and bone metastasis in breast and colorectal cancer via AKT and ERK signaling pathways (69-71) or through a mechanism involving AKT $/ \beta$-catenin/survivin in multiple myeloma (42). Our data demonstrate that KMT2A-R-induced AKT/ $\beta$-catenin/survivin is positively regulated by RUNX2. Mechanistically, we showed that RUNX2 regulates leukemic cell survival through direct transcriptional regulation of survivin and $\beta$-catenin. While $\beta$-catenin and survivin are both downstream targets of AKT signaling $(40,41)$, there is evidence that survivin is upregulated by active $\beta$-catenin (72). Importantly, $\beta$-catenin is required for leukemia initiation, survival, and proliferation of T-ALL (73-75) and KMT2A-R AML (76-79). Thus, we postulate that AKT/ $\beta$-catenin/survivin signaling serves as a functional regulator of RUNX2-mediated T-ALL cell survival. Targeting this pathway could be considered for therapeutic intervention in high-risk T-ALL (78-80).

Previous studies document the importance of CXCR4CXCL12 signaling in T-ALL cell migration, homing, leukemia development, and dissemination to the CNS (58-60). Our data revealed that CXCR4 is a direct transcriptional target for RUNX2 in T-ALL cells (Figure 5 and Supplemental Figure 4) and that its upregulation potentiates migration of T-ALL cells to CXCL12. Besides chemotaxis, CXCL12-CXCR4 signaling activates integrin receptors, which are essential for adhesion and transendothelial migration of lymphoblasts (57). Correspondingly, RUNX2 upregulation increases the levels of VLA-4 integrin and enhances T-ALL cell adhesion to fibronectin. Moreover, we show that RUNX2 induces critical mediators of focal adhesions, cell motility, and contractility, further supporting its role in promoting T-ALL migration (81-83). Collectively, we identify a mechanistic link between RUNX2 and reinforced CXCR4 signaling.

We showed that overexpression of RUNX2 exacerbates T-ALL progression, consistent with enhanced leukemia cell homing and infiltration of the BM and extramedullary sites, such as the meninges or liver (Figure 5). Importantly, these observations link RUNX2 upregulation with T-ALL progression and invasiveness. Consistently, aberrant RUNX2 expression has been associated with metastasis and disease progression in several solid tumors and hematological malignancies (27, 30-33, 36, 37, 66-68). Furthermore, studies by Stavropoulou et al. (56) elegantly showed that KMT2A-MLLT3 expression causes highly penetrant AML-expressing EMT genes, which we also found enriched in primary KMT2A-R T-ALL (14). In this study, we show that RUNX2 positively regulates critical EMT regulators, such as ZEB1, Snail, and vimentin (Figure 5). Given the roles of ZEB1 and other EMT proteins in progression of KMT2A-R AML and other tumors $(56,84)$, we hypothesize that enhanced T-ALL dissemination may be associated with RUNX2-mediated regulation of EMT. Notably, $\beta$-catenin and CXCR4, downstream targets of RUNX2 in our studies, are known to enhance EMT-like phenotype acquisition in invasive cancer cells $(85,86)$. Therefore, a possible role of RUNX2 in mediating acquisition of EMT gene signature through the modulation of $\beta$-catenin and CXCR 4 cannot be excluded. 
A
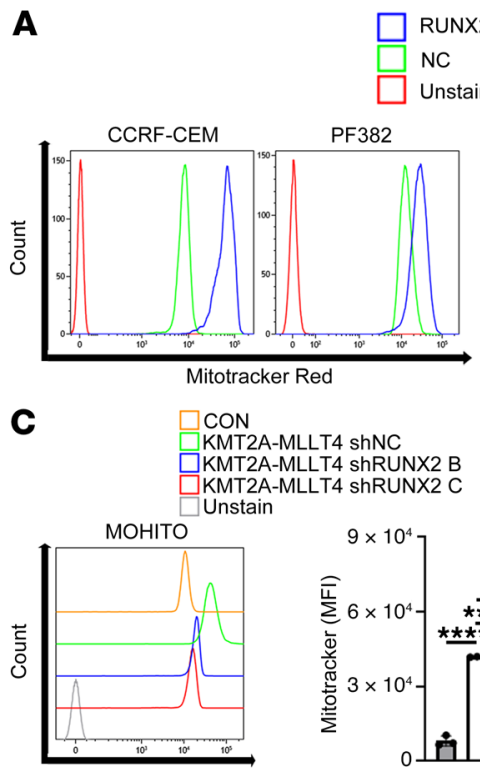

Mitotracker Red

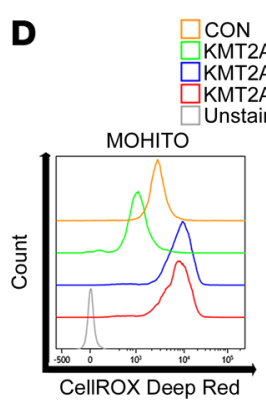

$\square$ CON

KMT2A-MLLT4 shNC

KMT2A-MLLT4 ShRUNX2 B Unstain
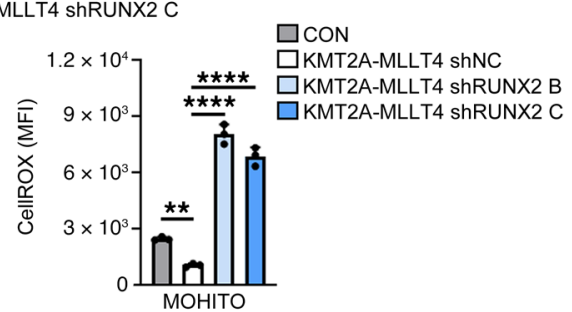

E

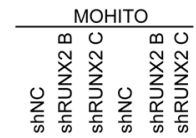

CCRF-CEM PF382

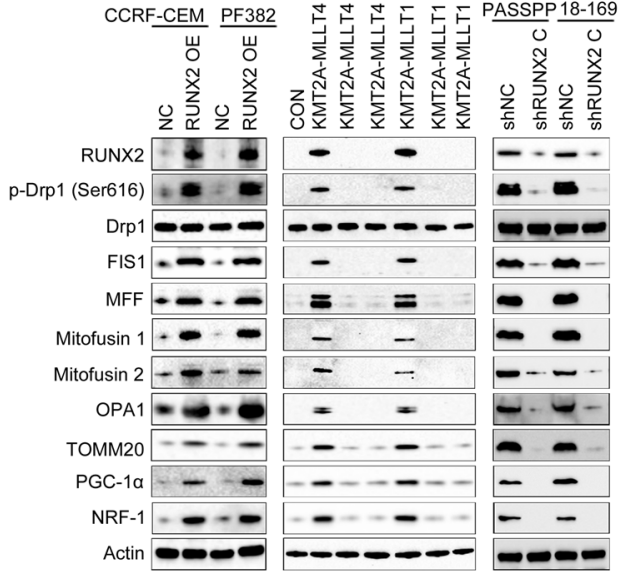

G

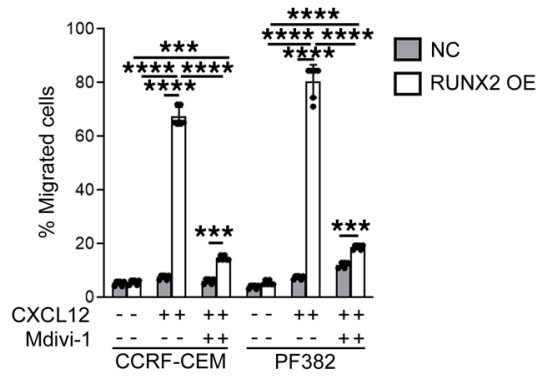

B

$\begin{array}{lll}\text { B } & \square \text { RUNX2 OE } & \square \text { NC } \\ & \square \text { NC } & \square \text { RUNX2 OE } \\ & \text { Unstain } & 6 \times 10^{3} 7^{* * * * * * * *}\end{array}$
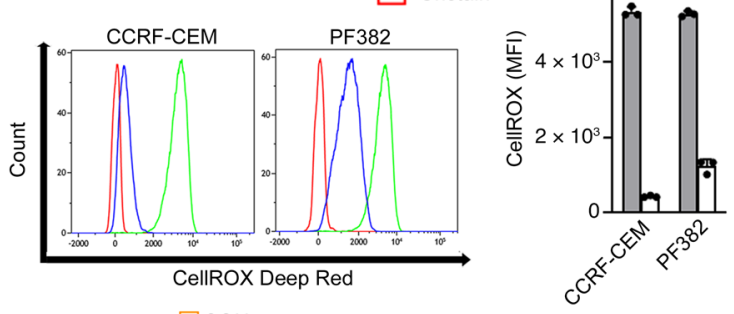

CON

KMT2A-MLLT1 shNC

KMT2A-MLLT1 shRUNX2 B
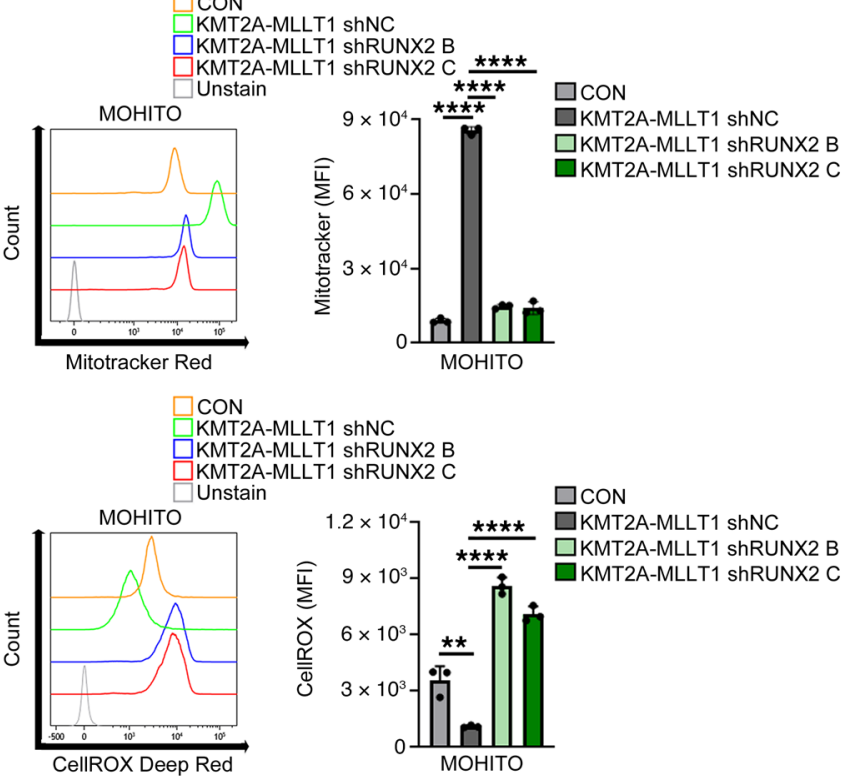

$\mathbf{F}$

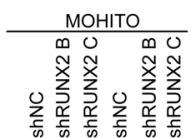

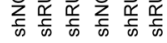

CCRF-CEM PF382

岁

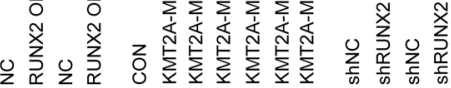

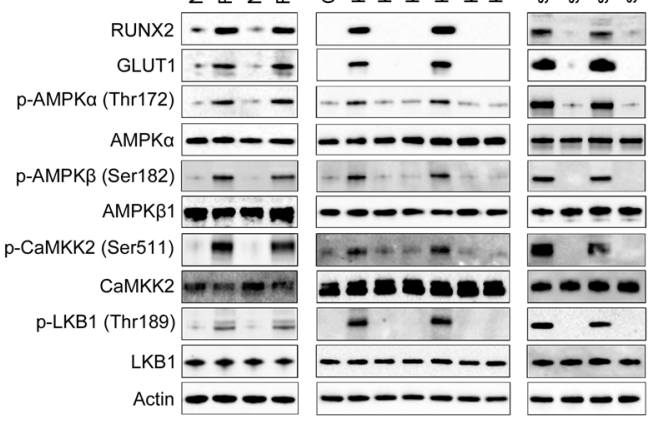

H

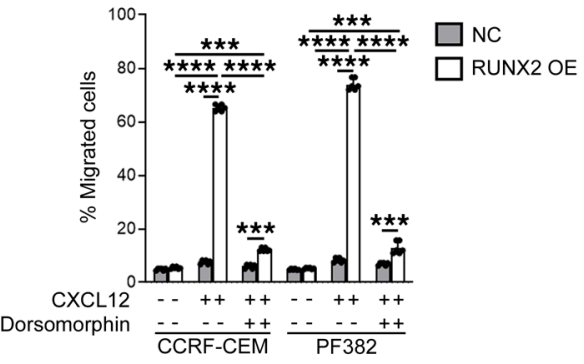


Figure 7. RUNX2 positively regulates mitochondrial dynamics and biogenesis. (A) Mitochondria membrane potential (MitoTracker Red CMXRos) and (B) ROS levels (CellROS Deep Red) were tested by flow cytometry in CCRF-CEM and PF382 cells transduced with RUNX2expressing (RUNX2 OE) and negative control plasmids. Representative histograms (left); MFI \pm SD, 3 separate experiments (right). (C) Mitochondrial membrane potential and (D) ROS production in MOHITO negative control cells and MOHITO-expressing KMT2A-R (KMT2A-MLLT4, KMT2AMLLT1) and transduced with RUNX2 shRNAs (shRUNX2 B and shRUNX2 C) or scrambled control. Representative histograms and MFI \pm SD, 3 independent experiments. (E and F) CCRF-CEM and PF382 cells with forced RUNX2 expression; MOHITO cells expressing control plasmid, KMT2A-R (KMT2A-MLLT1, KMT2A-MLLT4), and primary T-ALL cells (PASSPP, 18-169) were transduced with RUNX2 shRNAs and scrambled control. Representative Western blot for the expression of specified proteins $(n=2)$. (G and $\mathbf{H})$ Migration of CCRF-CEM and PF382 cells upon RUNX2 overexpression. Cells were pretreated with (G) a mitochondrial fission inhibitor, Mdivi-1 (1 $\mu \mathrm{M}, 16$ hours), or (H) AMPK pathway inhibitor, dorsomorphin ( $1 \mu \mathrm{M}, 16$ hours), followed by migration through the $3 \mu \mathrm{m}$ porous membrane in serum-free medium \pm CXCL12 ( $50 \mathrm{ng} / \mu \mathrm{l} ; 6$ hours). Data are shown as mean \pm SD, 3 separate experiments performed in duplicate. (A and B) Unpaired 2-tailed $t$ test with Holm-Šidák correction for multiple comparisons; (C and D) 1-way ANOVA with Tukey's multiple comparison test; (G and $\mathbf{H}$ ) 2-way ANOVA with Tukey's multiple comparison test. ${ }^{* *} P$ $<0.005 ;{ }^{* *} P<0.0005 ;{ }^{* * *} P<0.0001$.

In addition, we found increased levels of human TNF- $\alpha$ and IL-10 in serum of animals engrafted with RUNX2 OE cells. While TNF- $\alpha$ has been associated with leukemia initiation and progression (87), IL-10 is known to suppress antitumor immunity (88). Whether IL-10 and TNF- $\alpha$ modulate host immune response to facilitate T-ALL cell engraftment and proliferation remains unknown.

Most intriguingly, we found that RUNX2 significantly induces both glycolytic and oxidative metabolism corresponding to increased metabolic potential of T-ALL cells (Figure 6). This strongly suggests that RUNX2 enhances metabolic activity in T-ALL, a phenomenon seen in metastatic breast cancer, as opposed to the Warburg effect promoting glycolysis (89). Since RUNX2 exacerbates disease progression and extramedullary infiltration, we speculate that RUNX2-mediated interplay between glycolysis and mitochondrial respiration may define leukemic cell promigratory and invasive capability to target distinct organs and tissues. Further studies will determine whether and how metabolic reprogramming may affect leukemia site-specific infiltration and progression. As our results suggest, one such mechanism could be the direct transcriptional activation of metabolic regulators, such as PGK1, LDHA, and CHCHD2 by RUNX2.

T-ALL cells exhibit chronic metabolic stress and can adopt aerobic glycolysis for cell survival and disease progression (90, 91). However, AMPK activation can restrain aerobic glycolysis and promote oxidative phosphorylation to mitigate metabolic stress $(90,92)$. We found that RUNX2 induces AMPK activation in T-ALL (Figure 7), suggesting that oncogenic signaling in immature or KMT2A-R T-ALL may imbalance cell metabolism, leading to metabolic stress and RUNX2-mediated AMPK activation. An increase in mitochondrial membrane potential and reduced ROS levels in RUNX2 OE cells further suggest enhanced mitochondrial activity that leukemic cells might employ to meet metabolic demands related to proliferation, migration, or viability. Interestingly, AMPK is necessary to maintain leukemia-initiating cell survival by promoting GLUT1 expression in KMT2A-MLLT3 AML (93). In our studies, RUNX2 increased GLUT1 levels together with glucose uptake and glycolysis (Figure 6). Importantly, GLUT1 was identified as a direct transcriptional target of RUNX2 in differentiating osteoblasts (94). Therefore, this study identifies an important link between RUNX2 and AMPK in regulating balance between oxidative phosphorylation and glycolysis in T-ALL.

We demonstrated that RUNX2 modulates mitochondrial dynamics and biogenesis and that disruption of mitochondrial activity or AMPK signaling inhibits CXCR4-mediated T-ALL migration (Figure 7). Corresponding with previous studies indicating pivotal roles of mitochondrial fusion and fission in the regulation of cancer cell migration and invasiveness (95), our results raise the possibility that RUNX2 supports energy demands during cell migration by increasing mitochondrial bioenergetics and dynamics. For instance, Drp1, a master regulator of mitochondrial fission, is activated by RUNX2 and its pharmacological inhibition reduces T-ALL migration. Activation of Drp1 during RUNX2-induced chemotaxis indeed fits its essential role in T cell extravasation and homing into secondary lymphoid organs (96). Other studies showed that AMPK signaling induces downstream mediators of Drp1, MFF, or FIS1 in response to energy stress to support leukemic stem cell survival (97-99). Furthermore, mitochondrial fusion proteins are also upregulated by RUNX2 in our studies, and their aberrant expression was linked to metabolic reprograming in tumor metastasis $(95,100)$. Strikingly, we found that RUNX2 induces PGC1 $\alpha$, a central regulator of mitochondrial biogenesis and metabolism (101). Because AMPK induces mitochondrial biogenesis via PGC1 $\alpha$ in response to energy deprivation, this suggests a potential mechanism by which RUNX2-mediated metabolic stress induces AMPK-mediated upregulation of mitochondrial biogenesis. Our work identifies RUNX2 as a regulator of mitochondrial dynamics and biogenesis and suggests that targeting mitochondrial structures can be considered as an attractive strategy in eradicating leukemic cells.

As a proof of principle, we investigated the effect of pharmacological RUNX inhibition in T-ALL with elevated RUNX2. Others have already reported functional redundancy among RUNX proteins in myeloid KMT2A-R leukemia (62) and an oncogenic role for RUNX1 $(62,64)$ in KMT2A-R leukemia and in T-ALL (63). Our ChIP-Seq data showed RUNX2 binding and the presence of multiple RUNX consensus motifs at the promoters of RUNX1, RUNX2, and RUNX3 (Supplemental Table 4). Additionally, we observed Runx1 upregulation in Runx2-deficient KMT2A-MLLT1 transformed mouse BM progenitor cells. Therefore, we argued that allosteric inhibition of the RUNX-CBF $\beta$ interaction using the small molecule AI-10-104 (65) would be the most appropriate therapeutic strategy. We found that T-ALL cell lines and KMT2A-R patient-derived samples were equally sensitive to this compound, as previously reported for RUNX1-dependent T-ALL (Figure 8 and ref. 63). Importantly, gene set enrichment analysis confirmed that the gene-expression signatures of RUNX2-depleted and AI-10104-treated KARPAS-45 cells were highly similar. Interestingly, mitochondrial genes, such as MFN1 and UCP2, were downregulated along with many components of the electron transport chain (e.g., CYC1). In contrast, metallothionein genes were highly upregulated (Figure 8 and Supplemental Table 4). These data fur- 
A

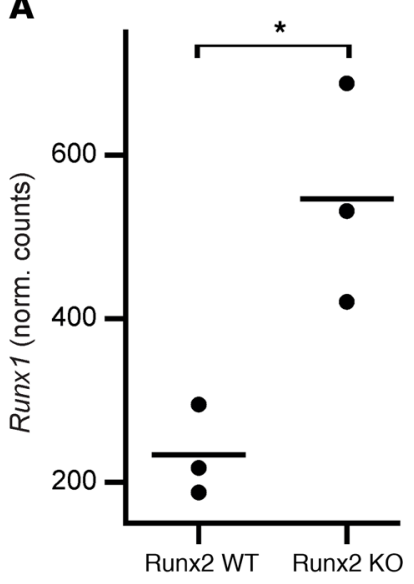

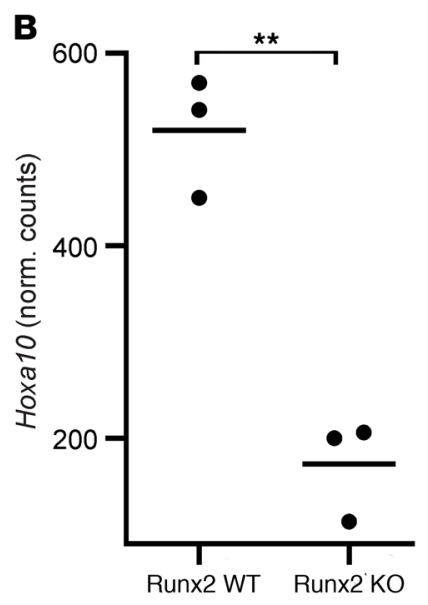

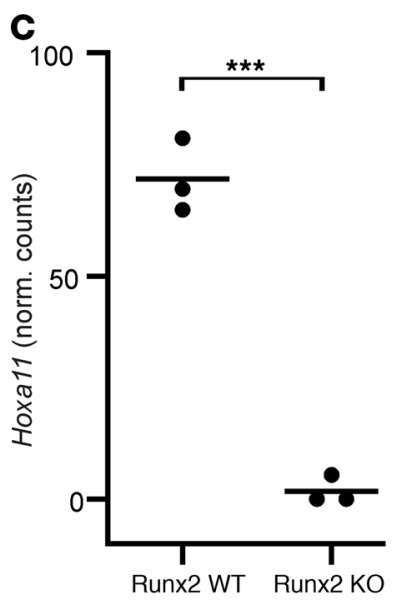

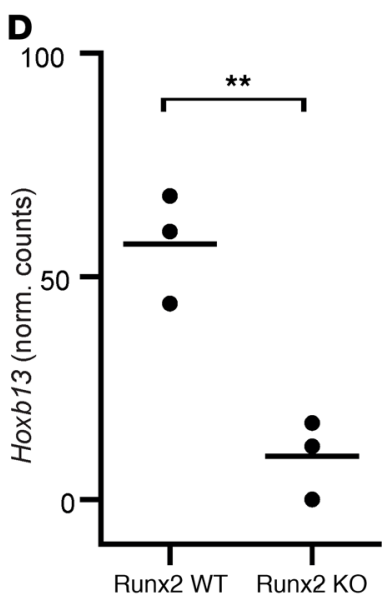

E
- $\operatorname{KARPAS}-45(12.98 \mu \mathrm{M})$
PER-117 (6.41 $\mu \mathrm{M})$
$\rightarrow \operatorname{LOUCY}(21.09 \mu \mathrm{M})$
$\rightarrow \operatorname{DND41}(12.53 \mu \mathrm{M})$
$\rightarrow \quad$ JURKAT $(12.52 \mu \mathrm{M})$

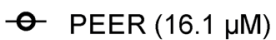
ఈ TALL-1 $(11.0 \mu \mathrm{M})$
$\triangle \quad \operatorname{ALL}-S I L(7.89 \mu \mathrm{M})$

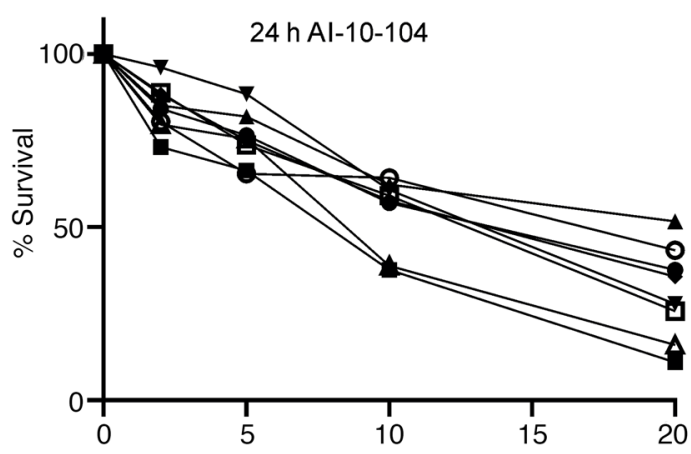

G

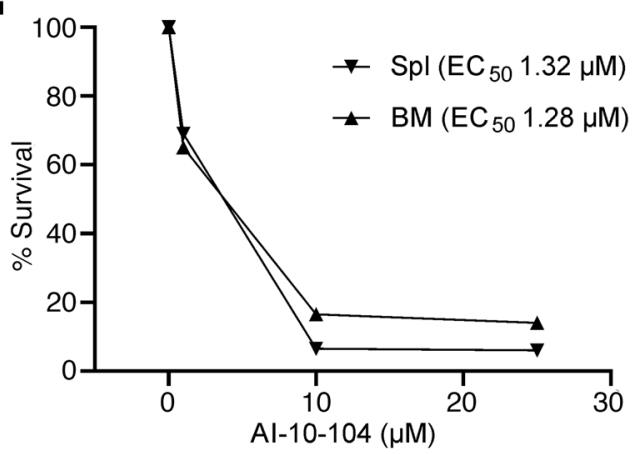

F

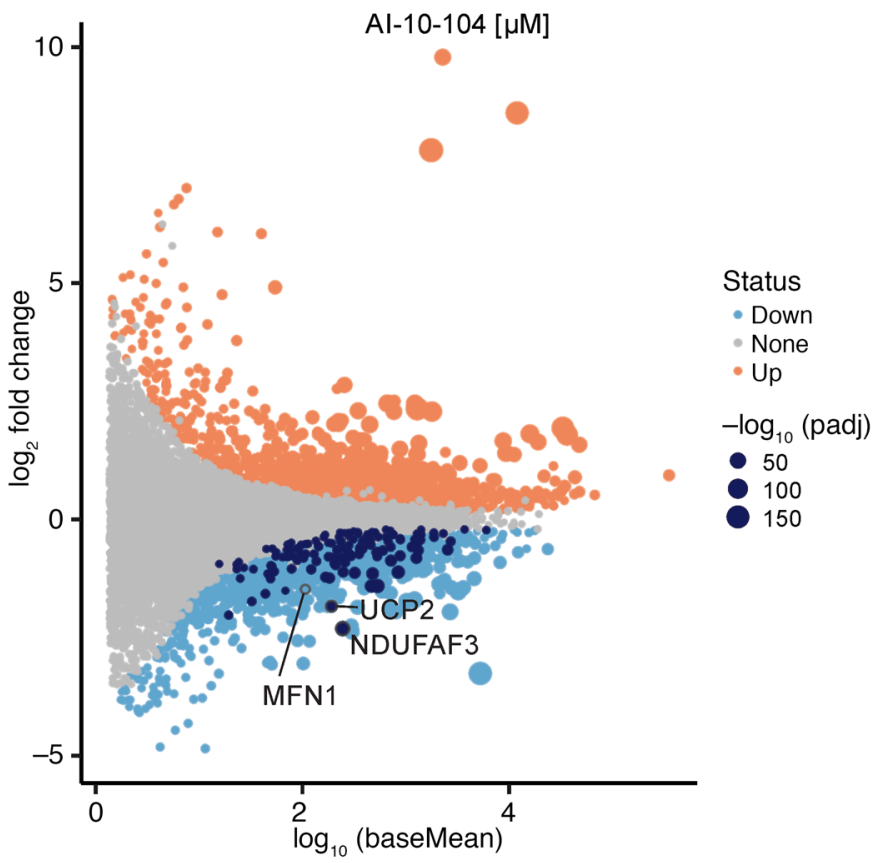

H

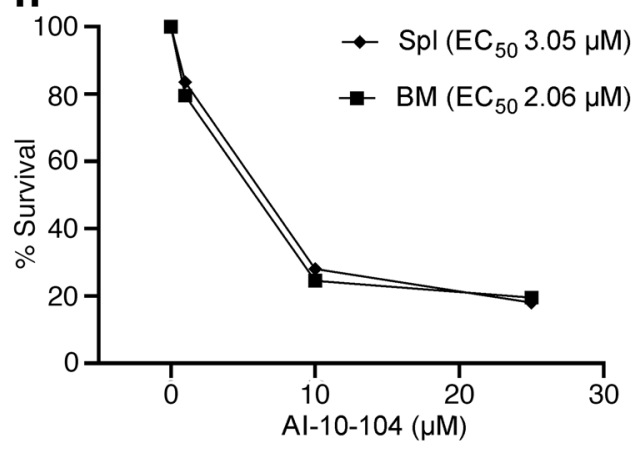

Figure 8. Immature T-ALL and KMT2A-rearranged leukemias are vulnerable to pharmacological targeting of the RUNX-CBF $\beta$ interaction. (A-D) Cene expression in KMT2A-MLLT1 transformed mouse (Runx $2^{f / f / f}$; Cre Ert2 ${ }^{\mathrm{tg} /+}$ ) BM progenitor cells. Full Runx2 KO was achieved by treatment of transduced progenitor cells with $400 \mathrm{nM}$ 4-hydroxytamoxifen for 24 hours prior to replating; vehicle-treated (EtOH) cells served as control (Runx2 WT). RNA-Seq normalized gene counts for (A) Runx1, (B) Hoxa10, (C) Hoxa11, and (D) Hoxb13. (E) A panel of T-ALL cell lines showed reduced survival in vitro upon treatment with Al-10-104 (Cell-Titer Glo assay). (F) MA plot showing significantly $\left(P_{\text {Adj }}<0.05\right)$ upregulated (red) and downregulated (blue) genes in KARPAS-45 upon 24-hour treatment with $20 \mu \mathrm{M} \mathrm{Al}-10-104$ compared with DMSO controls. Enriched gene set of mitochondrial inner membrane (MIM) genes represented in dark blue. Data based on 4 independent biological replicates. PDX samples from KMT2A-MLLT1 (G) and KMT2A-MLLT4 (H) patients showed reduced survival upon 24-hour treatment with Al-10-104. (A-D) Unpaired 2-tailed $t$ test. ${ }^{*} P<0.05 ;{ }^{* *} P<0.005 ;{ }^{* * *} P<0.0005$. 
ther support our findings that RUNX2 mediates mitochondrial metabolism and dynamics and that RUNX2 targeting leads to metabolic stress in KMT2A-R and immature T-ALL. While further improvements in potency and stability of the tested inhibitors are needed to allow their efficacious use in vivo (102), our results further advocate the RUNX-CBF $\beta$ interaction as a promising, druggable target in leukemia.

In summary, we show that RUNX2 is aberrantly expressed in a subset of high-risk T-ALL patients and is required for T-ALL survival. These studies establish RUNX2 as a dependency factor in high-risk T-ALL and implicate RUNX2 upregulation as a critical mediator of disease progression. We uncover a potentially novel function of RUNX2 in regulating leukemic cell metabolism and mitochondrial dynamics. In addition, we identify RUNX2 and its downstream targets for therapeutic intervention in immature and KMT2A-R T-ALL.

\section{Methods.}

For further information, see Supplemental Methods.

Data availability. All original microarray data were deposited in the NCBI's Gene Expression Omnibus database (GEO GSE151823).

Statistics. The statistical analyses were performed using GraphPad Prism 9. Results were considered statistically significant at $P<0.05$. The appropriate statistical tests are specified in the figure legends.

Study approval. Samples were obtained from patients enrolled in COG T-ALL study AALLO434 (16) at the University of New Mexico (IRB approval nos. 16-246 and 03-183) or at Ghent University Hospital (ethical committee approval no. 15/87). All patients or their parents or guardians provided written, informed consent in accordance with the Declaration of Helsinki and local institutional guidelines. The ethical committees on animal welfare at the University of New Mexico (19-30020-HSC) and Ghent University Hospital approved all animal experiments.

\section{Author contributions}

NDS, FM, PVV, and KMW designed the studies. NDS, FM, PVV, KMW, CKN, MN, DRP, LD, SP, TP, LR, BL, SG, FVN, DLD, and
SDM collected and assembled data. YY, TT, MDPR, SVC, LCC, and RSK provided critical reagents and the mouse model. CGM, SSW, MLL, SPH, BDM, TL, FWVD, and RBL provided patient samples and clinical data. NDS, FM, PVV, KMW, JR, WVL, WO, EFC, LAS, AC, HK, DRP, and SDM analyzed and interpreted data. KMW, FM, and NDS wrote the manuscript. KMW and PVV originated and supervised the project. All authors reviewed and approved the manuscript. FM and NDS contributed equally to this work and are listed in alphabetical order in the author list.

\section{Acknowledgments}

This project was supported by NIH National Cancer Institute (NCI) grant R01 CA237165 (to KMW). Other grant support is as follows: NIH National Center for Advancing Translational Sciences (NCATS) Clinical and Translational Science Awards (CTSA) (8UL1TR000041), NIH NCI Cancer Center Support Grant (P30CA118100), University of New Mexico Autophagy, Inflammation \& Metabolism Center (AIM) cores (P20GM121176), U24 CA114766 (Children's Oncology Group [COG] Specimen Banking), R35 CA197695 (to CGM), the Research Foundation Flanders (FWO), the Flemish Agency for Innovation by Science and Technology and Stand up to Cancer (the Flemish Cancer Society), the National Health and Medical Research Council of Australia (APP1142627 to RSK; APP1059804 and APP1157871 to RBL), and Kinderkankerfonds (to TL).We acknowledge the Shared Flow Cytometry Resource, the Animal Models Shared Resource, the Animal Resource Facility, and the Autophagy, Inflammation and Metabolism Center of the University of New Mexico. We thank Irina Lagutina for technical assistance. We acknowledge the COG Cell Bank (AALL15B1-Q, Matlawska) and EORTC-58081-CLG (NCT01185886, Van Vlierberghe) for provision of patient samples.

Address correspondence to: Ksenia Matlawska-Wasowska, Department of Pediatrics, Division of Hematology-Oncology, University of New Mexico, Albuquerque, New Mexico 87131, USA. Phone: 1.505.272.6177; Email: KMatlawska-Wasowska@salud.unm.edu.
1. Pui CH, et al. Acute lymphoblastic leukaemia. Lancet. 2008;371(9617):1030-1043.

2. Winter SS, et al. Improved survival for children and young adults with t-lineage acute lymphoblastic leukemia: results from the children's oncology group AALL0 434 methotrexate randomization. J Clin Oncol. 2018;36(29):2926-2934.

3. Armstrong SA, et al. MLL translocations specify a distinct gene expression profile that distinguishes a unique leukemia. Nat Genet. 2002;30(1):41-47.

4. Ferrando AA, et al. Gene expression signatures define novel oncogenic pathways in $\mathrm{T}$ cell acute lymphoblastic leukemia. Cancer Cell. 2002;1(1):75-87.

5. Homminga I, et al. Integrated transcript and genome analyses reveal NKX2-1 and MEF2C as potential oncogenes in T cell acute lymphoblastic leukemia. Cancer Cell. 2011;19(4):484-497.

6. Soulier J, et al. HOXA genes are included in genetic and biologic networks defining human acute T-cell leukemia (T-ALL). Blood. 2005;106(1):274-286.
7. Van Vlierberghe P, Ferrando A. The molecular basis of T cell acute lymphoblastic leukemia. J Clin Invest. 2012;122(10):3398-3406.

8. Liu Y, et al. The genomic landscape of pediatric and young adult T-lineage acute lymphoblastic leukemia. Nat Genet. 2017;49(8):1211-1218.

9. Zuurbier L, et al. Immature MEF2C-dysregulated T-cell leukemia patients have an early T-cell precursor acute lymphoblastic leukemia gene signature and typically have non-rearranged T-cell receptors. Haematologica. 2014;99(1):94-102.

10. Coustan-Smith E, et al. Early T-cell precursor leukaemia: a subtype of very high-risk acute lymphoblastic leukaemia. Lancet Oncol. 2009;10(2):147-156.

11. Inukai $\mathrm{T}$, et al. Clinical significance of early T-cell precursor acute lymphoblastic leukaemia: results of the Tokyo Children's Cancer Study Group Study L99-15. Br J Haematol. 2012;156(3):358-365.

12. Van Vlierberghe $P$, et al. Prognostic relevance of integrated genetic profiling in adult T-cell acute lymphoblastic leukemia. Blood. 2013;122(1):74-82.

13. Ferrando AA, et al. Gene expression signatures in MLL-rearranged T-lineage and B-precursor acute leukemias: dominance of HOX dysregulation. Blood. 2003;102(1):262-268

14. Kang H, et al. Dysregulated transcriptional networks in KMT2A- and MLLT1O-rearranged T-ALL. Biomark Res. 2018;6:27.

15. Winters AC, Bernt KM. MLL-rearranged leukemias-an update on science and clinical approaches. Front Pediatr. 2017;5:4.

16. Matlawska-Wasowska K, et al. MLL rearrangements impact outcome in HOXA-deregulated T-lineage acute lymphoblastic leukemia: a Children's Oncology Group Study. Leukemia. 2016;30(9):1909-1912.

17. Peterson JF, et al. KMT2A (MLL) rearrangements observed in pediatric/young adult T-lymphoblastic leukemia/lymphoma: A 10-year review from a single cytogenetic laboratory. Genes Chromosomes Cancer. 2018;57(11):541-546. 
18. Blyth K, et al. The RUNX genes: gain or loss of function in cancer. Nat Rev Cancer. 2005;5(5):376-387.

19. Ito Y, et al. The RUNX family: developmental regulators in cancer. Nat Rev Cancer. 2015;15(2):81-95.

20. Warren AJ, et al. Structural basis for the heterodimeric interaction between the acute leukaemiaassociated transcription factors AML1 and CBFbeta. EMBO J. 2000;19(12):3004-3015.

21. Growney JD, et al. Loss of Runx1 perturbs adult hematopoiesis and is associated with a myeloproliferative phenotype. Blood. 2005;106(2):494-504.

22. Okuda T, et al. AML1, the target of multiple chromosomal translocations in human leukemia, is essential for normal fetal liver hematopoiesis. Cell. 1996;84(2):321-330.

23. Ducy P, et al. Osf2/Cbfa1: a transcriptional activator of osteoblast differentiation. Cell. 1997;89(5):747-754.

24. Enomoto $\mathrm{H}$, et al. $\mathrm{Cbfa} 1$ is a positive regulatory factor in chondrocyte maturation. J Biol Chem. 2000;275(12):8695-8702.

25. Komori T, et al. Targeted disruption of Cbfa1 results in a complete lack of bone formation owing to maturational arrest of osteoblasts. Cell. 1997;89(5):755-764.

26. Otto F, et al. Cbfa1, a candidate gene for cleidocranial dysplasia syndrome, is essential for osteoblast differentiation and bone development. Cell. 1997;89(5):765-771.

27. Kuo YH, et al. Runx2 induces acute myeloid leukemia in cooperation with Cbfbeta-SMMHC in mice. Blood. 2009;113(14):3323-3332.

28. Vaillant F, et al. Enforced expression of Runx2 perturbs T cell development at a stage coincident with beta-selection. J Immunol. 2002;169(6):2866-2874.

29. Lau CC, et al. Frequent amplification and rearrangement of chromosomal bands 6p12-p21 and 17 p11.2 in osteosarcoma. Genes Chromosomes Cancer. 2004;39(1):11-21.

30. Martin JW, et al. The role of RUNX2 in osteosarcoma oncogenesis. Sarcoma. 2011;2011:282745.

31. Sadikovic B, et al. Expression analysis of genes associated with human osteosarcoma tumors shows correlation of RUNX2 overexpression with poor response to chemotherapy. BMC Cancer. 2010;10:202.

32. Akech J, et al. Runx2 association with progression of prostate cancer in patients: mechanisms mediating bone osteolysis and osteoblastic metastatic lesions. Oncogene. 2010;29(6):811-821.

33. Pratap J, et al. Runx 2 transcriptional activation of Indian Hedgehog and a downstream bone metastatic pathway in breast cancer cells. Cancer Res. 2008;68(19):7795-7802.

34. Stewart M, et al. Proviral insertions induce the expression of bone-specific isoforms of PEBP2alphaA (CBFA1): evidence for a new myc collaborating oncogene. Proc Natl Acad Sci US A. 1997;94(16):8646-8651.

35. Vaillant F, et al. A full-length Cbfa1 gene product perturbs T-cell development and promotes lymphomagenesis in synergy with myc. Oncogene. 1999;18(50):7124-7134

36. Blyth K, et al. Runx2 and MYC collaborate in lym- phoma development by suppressing apoptotic and growth arrest pathways in vivo. Cancer Res. 2006;66(4):2195-2201.

37. Kubota S, et al. Lineage-specific RUNX2 super-enhancer activates MYC and promotes the development of blastic plasmacytoid dendritic cell neoplasm. Nat Commun. 2019;10(1):1653.

38. Clappier E, et al. Clonal selection in xenografted human $\mathrm{T}$ cell acute lymphoblastic leukemia recapitulates gain of malignancy at relapse. JExp Med. 2011;208(4):653-661.

39. Takarada T, et al. An analysis of skeletal development in osteoblast-specific and chondrocytespecific runt-related transcription factor-2 (Runx2) knockout mice. J Bone Miner Res. 2013;28(10):2064-2069.

40. Fang D, et al. Phosphorylation of $\beta$-catenin by AKT promotes beta-catenin transcriptional activity. J Biol Chem. 2007;282(15):11221-11229.

41. Hideshima T, et al. Inhibition of Akt induces significant downregulation of survivin and cytotoxicity in human multiple myeloma cells. Br J Haematol. 2007;138(6):783-791.

42. Trotter TN, et al. Myeloma cell-derived Runx2 promotes myeloma progression in bone. Blood. 2015;125(23):3598-3608.

43. Wilson NK, et al. Combinatorial transcriptional control in blood stem/progenitor cells: genomewide analysis of ten major transcriptional regulators. Cell Stem Cell. 2010;7(4):532-544.

44. Sanda T, et al. Core transcriptional regulatory circuit controlled by the TAL1 complex in human $\mathrm{T}$ cell acute lymphoblastic leukemia. Cancer Cell. 2012;22(2):209-221.

45. McLean CY, et al. GREAT improves functional interpretation of cis-regulatory regions. $\mathrm{Nat}$ Biotechnol. 2010;28(5):495-501.

46. Herranz D, et al. A NOTCH1-driven MYC enhancer promotes T cell development, transformation and acute lymphoblastic leukemia. Nat Med. 2014;20(10):1130-1137.

47. Kerry J, et al. MLL-AF4 spreading identifies binding sites that are distinct from super-enhancers and that govern sensitivity to DOT1L inhibition in leukemia. Cell Rep. 2017;18(2):482-495.

48. Kohlmann A, et al. New insights into MLL gene rearranged acute leukemias using gene expression profiling: shared pathways, lineage commitment, and partner genes. Leukemia. 2005;19(6):953-964.

49. Gu Z, et al. PAX5-driven subtypes of B-progenitor acute lymphoblastic leukemia. Nat Genet. 2019;51(2):296-307.

50. Balgobind BV, et al. Evaluation of gene expression signatures predictive of cytogenetic and molecular subtypes of pediatric acute myeloid leukemia. Haematologica. 2011;96(2):221-230

51. Milne TA, et al. Leukemogenic MLL fusion proteins bind across a broad region of the Hox a9 locus, promoting transcription and multiple histone modifications. Cancer Res. 2005;65(24):11367-11374.

52. Lin S, et al. Instructive role of MLL-fusion proteins revealed by a model of $\mathrm{t}(4 ; 11)$ pro-B acute lymphoblastic leukemia. Cancer Cell. 2016;30(5):737-749.

53. Bernt KM, et al. MLL-rearranged leukemia is dependent on aberrant H3K79 methylation by
DOT1L. Cancer Cell. 2011;20(1):66-78.

54. Garcia-Cuellar MP, et al. Leukemogenic MLLENL fusions induce alternative chromatin states to drive a functionally dichotomous group of target genes. Cell Rep. 2016;15(2):310-322.

55. Guenther MG, et al. Aberrant chromatin at genes encoding stem cell regulators in human mixed-lineage leukemia. Genes Dev. 2008;22(24):3403-3408.

56. Stavropoulou V, et al. MLL-AF9 expression in hematopoietic stem cells drives a highly invasive AML expressing EMT-related genes linked to poor outcome. Cancer Cell. 2016;30(1):43-58.

57. Shen $\mathrm{W}$, et al. The chemokine receptor CXCR4 enhances integrin-mediated in vitro adhesion and facilitates engraftment of leukemic precursor-B cells in the bone marrow. Exp Hematol. 2001;29(12):1439-1447.

58. Passaro D, et al. CXCR4 is required for leukemia-initiating cell activity in T cell acute lymphoblastic leukemia. Cancer Cell. 2015;27(6):769-779.

59. Pitt LA, et al. CXCL12-producing vascular endothelial niches control acute $\mathrm{T}$ cell leukemia maintenance. Cancer Cell. 2015;27(6):755-768.

60. Jost TR, et al. Role of CXCR4-mediated bone marrow colonization in CNS infiltration by $\mathrm{T}$ cell acute lymphoblastic leukemia. J Leukoc Biol. 2016;99(6):1077-1087.

61. Herzig S, Shaw RJ. AMPK: guardian of metabolism and mitochondrial homeostasis. Nat Rev Mol Cell Biol. 2018;19(2):121-135.

62. Goyama S, et al. Transcription factor RUNX1 promotes survival of acute myeloid leukemia cells. J Clin Invest. 2013;123(9):3876-3888.

63. Choi A, et al. RUNX1 is required for oncogenic $M y b$ and $M y c$ enhancer activity in T-cell acute lymphoblastic leukemia. Blood. 2017;130(15):1722-1733.

64. Wilkinson AC, et al. RUNX1 is a key target in $\mathrm{t}(4 ; 11)$ leukemias that contributes to gene activation through an AF4-MLL complex interaction. Cell Rep. 2013;3(1):116-127.

65. Illendula A, et al. Small molecule inhibitor of CBFbeta-RUNX binding for RUNX transcription factor driven cancers. EBioMedicine. 2016;8:117-131.

66. Vladimirova $\mathrm{V}$, et al. Runx 2 is expressed in human glioma cells and mediates the expression of galectin-3. J Neurosci Res. 2008;86(11):2450-2461.

67. Li XQ, et al. RUNX2 promotes breast cancer bone metastasis by increasing integrin $\alpha 5$-mediated colonization. Cancer Lett. 2016;380(1):78-86.

68. Guo ZJ, et al. Transcription factor RUNX2 upregulates chemokine receptor CXCR4 to promote invasive and metastatic potentials of human gastric cancer. Oncotarget. 2016;7(15):20999-21012.

69. Tandon M, et al. Role of Runx2 in IGF-1R $\beta$ / Akt- and AMPK/Erk-dependent growth, survival and sensitivity towards metformin in breast cancer bone metastasis. Oncogene. 2016;35(36):4730-4740.

70. Tandon M, et al. Role of Runx2 in crosstalk between Mek/Erk and PI3K/Akt signaling in MCF-10A cells. J Cell Biochem. 2014;115(12):2208-2217.

71. Tandon M, et al. Runx2 activates PI3K/Akt 
signaling via mTORC2 regulation in invasive breast cancer cells. Breast Cancer Res. 2014;16(1):R16.

72. Tapia JC, et al. Casein kinase 2 (CK2) increases survivin expression via enhanced beta-catenin$\mathrm{T}$ cell factor/lymphoid enhancer binding factor-dependent transcription. Proc Natl Acad SciU S A. 2006;103(41):15079-15084.

73. Gekas $C$, et al. $\beta$-Catenin is required for T-cell leukemia initiation and MYC transcription downstream of Notch1. Leukemia. 2016;30(10):2002-2010.

74. Kaveri D, et al. $\beta$-Catenin activation synergizes with Pten loss and Myc overexpression in Notch-independent T-ALL. Blood. 2013;122(5):694-704.

75. Giambra V, et al. Leukemia stem cells in T-ALL require active Hif1 $\alpha$ and Wnt signaling. Blood. 2015;125(25):3917-3927.

76. Wang Y, et al. The Wnt/beta-catenin pathway is required for the development of leukemia stem cells in AML. Science. 2010;327(5973):1650-1653.

77. Yeung J, et al. $\beta$-Catenin mediates the establishment and drug resistance of MLL leukemic stem cells. Cancer Cell. 2010;18(6):606-618.

78. Park E, et al. Targeting survivin overcomes drug resistance in acute lymphoblastic leukemia. Blood.2011;118(8):2191-2199.

79. Tyner JW, et al. Targeting survivin and $\mathrm{p} 53$ in pediatric acute lymphoblastic leukemia. Leukemia. 2012;26(4):623-632.

80. Evangelisti C, et al. Targeting Wnt/ $\beta$-catenin and PI3K/Akt/mTOR pathways in T-cell acute lymphoblastic leukemia. J Cell Physiol. 2020;235(6):5413-5428.

81. Lopez-Colome AM, et al. Paxillin: a crossroad in pathological cell migration.J Hematol Oncol. 2017;10(1):50.

82. Glodek AM, et al. Focal adhesion kinase is required for CXCL12-induced chemotactic and pro-adhesive responses in hematopoietic precursor cells. Leukemia. 2007;21(8):1723-1732.

83. Clucas J, Valderrama F. ERM proteins in cancer progression. J Cell Sci. 2014;127(Pt 2):267-275.

84. Soen B, et al. ZEB proteins in leukemia: friends, foes, or friendly foes? Hemasphere. 2018;2(3):e43.

85. Sanchez-Tillo E, et al. $\beta$-catenin/TCF 4 complex induces the epithelial-to-mesenchymal transition (EMT)-activator ZEB1 to regulate tumor invasiveness. Proc Natl Acad Sci U S A. 2011;108(48):19204-19209.

86. Roccaro AM, et al. CXCR4 regulates extramedullary myeloma through epithelial-mesenchymal-transition-like transcriptional activation. Cell Rep. 2015;12(4):622-635.

87. Kagoya Y, et al. Positive feedback between NF-кB and TNF- $\alpha$ promotes leukemia-initiating cell capacity. JClin Invest. 2014;124(2):528-542.

88. Alhakeem SS, et al. Chronic lymphocytic leukemiaderived IL-10 suppresses antitumor immunity. JImmunol. 2018;200(12):4180-4189.

89. Dupuy F, et al. PDK1-dependent metabolic reprogramming dictates metastatic potential in breast cancer. Cell Metab. 2015;22(4):577-589.

90. Kishton RJ, et al. AMPK is essential to balance glycolysis and mitochondrial metabolism to control T-ALL cell stress and survival. Cell Metab. 2016;23(4):649-662.

91. Herranz D, et al. Metabolic reprogramming induces resistance to anti-NOTCH1 therapies in T cell acute lymphoblastic leukemia. Nat Med. 2015;21(10):1182-1189.

92. Faubert B, et al. AMPK is a negative regulator of the Warburg effect and suppresses tumor growth in vivo. Cell Metab. 2013;17(1):113-124.

93. Saito Y, et al. AMPK protects leukemiainitiating cells in myeloid leukemias from metabolic stress in the bone marrow. Cell Stem Cell. 2015;17(5):585-596.

94. Wei J, et al. Glucose uptake and Runx2 synergize to orchestrate osteoblast differentiation and bone formation. Cell. 2015;161(7):1576-1591.

95. Zhang Z, et al. MFN1-dependent alteration of mitochondrial dynamics drives hepatocellular carcinoma metastasis by glucose metabolic reprogramming. Br JCancer. 2020;122(2):209-220.

96. Simula L, et al. Drp1 controls effective T cell immune-surveillance by regulating $\mathrm{T}$ cell migration, proliferation, and cMyc-dependent metabolic reprogramming. Cell Rep. 2018;25(11):3059-3073.

97. Pei S, et al. AMPK/FIS1-mediated mitophagy is required for self-renewal of human AML stem cells. Cell Stem Cell. 2018;23(1):86-100.

98. Toyama EQ, et al. Metabolism. AMP-activated protein kinase mediates mitochondrial fission in response to energy stress. Science. 2016;351(6270):275-281.

99. Cai J, et al. ERK/Drp1-dependent mitochondrial fission is involved in the MSC-induced drug resistance of T-cell acute lymphoblastic leukemia cells. Cell Death Dis. 2016;7(11):e2459.

100.Yu M, et al. Mitochondrial fusion exploits a therapeutic vulnerability of pancreatic cancer. JCI Insight. 2019;5:126915.

101.LeBleu VS, et al. PGC-1 $\alpha$ mediates mitochondrial biogenesis and oxidative phosphorylation in cancer cells to promote metastasis. Nat Cell Biol. 2014;16(10):992-1003, 1-15.

102.Carlton AL, et al. Small molecule inhibition of the CBFß/RUNX interaction decreases ovarian cancer growth and migration through alterations in genes related to epithelial-to-mesenchymal transition. Gynecol Oncol. 2018;149(2):350-360.

103. Bene MC, et al. Proposals for the immunological classification of acute leukemias. European Group for the Immunological Characterization of Leukemias (EGIL). Leukemia. 1995;9(10):1783-1786. 